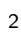
4 (1) 5

\title{
Pareto optimality solution of the multi-objective photogrammetric resection-intersection problem
}

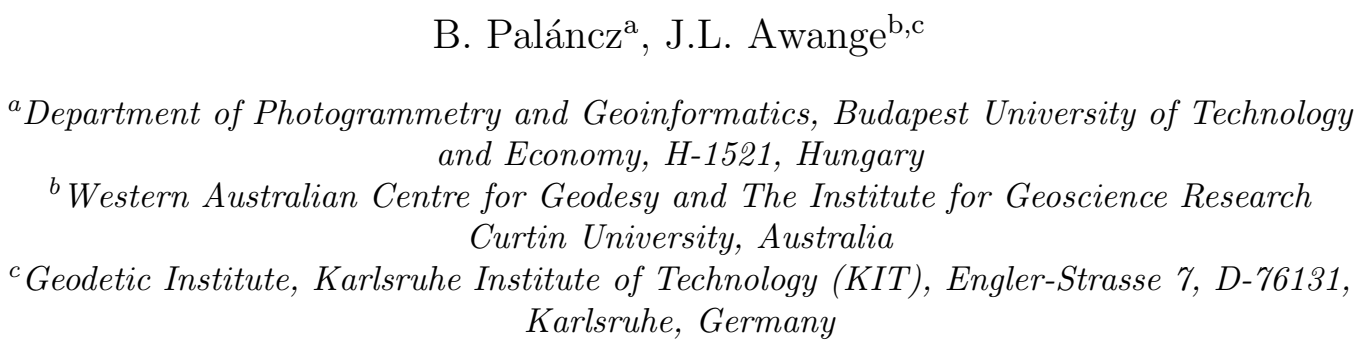

\section{Abstract}

Reconstruction of architectural structures from photographs has recently experienced intensive efforts in computer vision research. This is achieved through the solution of nonlinear least squares (NLS) problems to obtain accurate structure and motion estimates. In Photogrammetry, NLS contribute to the determination of the 3 -dimensional (3D) terrain models from the images taken from photographs. The traditional NLS approach for solving the resection-intersection problem based on implicit formulation on the one hand suffers from the lack of provision by which the involved variables can be weighted. On the other hand, incorporation of explicit formulation expresses the objectives to be minimized in different forms, thus resulting in different parametric values for the estimated parameters at non-zero residuals. Sometimes, these objectives may conflict in a Pareto sense, namely, a small change in the parameters results in the increase of one objective and a decrease of the other, as is often the case in multi-objective problems. Such is often the case with error - in - all - variable (EIV) models, e.g., in the 
resection-intersection problem where such change in the parameters could be caused by errors in both image and reference coordinates. This study proposes the Pareto optimal approach as a possible improvement to the solution of the resection-intersection problem, where it provides simultaneous estimation of the coordinates and orientation parameters of the cameras in a two or multistation camera system on the basis of a properly weighted multiobjective function. This objective represents the weighted sum of the square of the direct explicit differences of the measured and computed ground as well as the image coordinates. The effectiveness of the proposed method is demonstrated by two camera calibration problems, where the internal and external orientation parameters are estimated on the basis of the collinearity equations, employing the data of a Manhattan-type test field as well as the data of an outdoor, real case experiment. In addition, an architectural structural reconstruction of the Merton college court in Oxford (UK) via estimation of camera matrices is also presented. Although these two problems are different, where the first case considers the error reduction of the image and spatial coordinates, while the second case considers the precision of the space coordinates, the Pareto optimality can handle both problems in a general and flexible way.

Keywords: Pareto optimality, photogrammetric positioning, resection-intersection, symbolic-numeric solution, Pareto-front, multi-objective optimization 


\section{Introduction}

In computer vision and model-based vision, resection-intersection technique (Chen and Medioni 1999, Mahamud et al. 2000, Triggs et al. 2001) is often used to perform adjustment that plays an essential role in obtaining accurate structure and motion estimates (see, e.g., Bartoli 2002; Olsonn et al. 2009), while in photogrammetry, it is used to perform bundle adjustment to obtain a 3-dimensional (3D) terrain models from images taken from photographs. Indeed, in recent years, the demand for realistic reconstruction and modeling of objects and human bodies is increasing both for animation and medical applications (e.g., Remondino 2002). For example, the significant role played by resection and intersection is discussed e.g., in Börlin (2002), where resection methods is applied in radiostereometric analysis (RSA) to reconstruct the projection geometries, while the intersection technique is used to reconstruct the 3D-coordinates of the patient markers. Radiostereometric analysis has been widely used in orthopaedics for studying, e.g., prosthetic implant migration and wear, joint stability and kinematics, bone growth, and fracture healing (Börlin 2002). These applications of resection-intersection method, just to list but a few, underscores the need for further improvements and refinements of the existing techniques, and also testing others that could offer more flexibility and optimum results.

Generally, in order to determine the $3 \mathrm{D}$ position $(X, Y, Z)$ of a point in space (e.g., the 3-dimensional (3D) coordinates of the patient markers) through intersection, at least two photo images of the point are required with coordinates $(x, y)$ on each of the photo planes. In addition to these coordinates, carrying out intersection requires one to know the orientation 
parameters of the two cameras, which is often solved through resection. For resection, the internal and external orientation parameters of a camera model are determined based on the collinearity equations (see e.g. Mikhail et al. 2001; Forsyth and Ponce 2003; Awange and Kiema 2013). In computer vision, the problem of the determination of the exterior orientation parameters is known as the pose estimation problem (see, e.g., Grussenmeyer and Al Khalil 2002). Grussenmeyer and Al Khalil (2002) present a survey of methods for the determination of the exterior parameters in photogrammetry and classify them into three groups; approximate methods, the standard point-based methods derived from collinearity, coplanarity or co-angularity conditions, and the orientation methods based on constraints and projective geometry concepts (e.g., Grafarend and Shan 1997a,b).

There exists several methods for solving the combined resection-intersection problem, e.g., Grafarend and Shan (1997c) who present an algorithmic based on Möbius barycentric coordinates and Bartoli (2002) who adapt a quasilinear optimizations that uses the original cost function of bundle adjustment, which preserves optimality, and handle a great variety of camera models in a unified manner. Most frequently used methods to solve resection problem, however, are the different variants of the direct linear transformation (DLT), see e.g. Young-Hoo Kwon (1998) and Hartley and Zisserman (2003). In certain simplified cases, even symbolic or semi-symbolic solutions can be given, see e.g., Ameller et al. (2000), Awange and Grafarend (2005) and Awange et al. (2010). However, all of these DLT methods have three common features (see e.g., Atkinson 1996), namely (i) the orientation parameters of each camera are 
estimated independently through resection but the positions determination using intersection uses all of the orientation and image coordinates simultaneously, (ii) the equations used for parameter estimation contain the measured coordinates implicitly, which means that the resulting residuals have no physical interpretation, and (iii) because of this implicit formulation, neither the reference nor the measured image coordinates can be weighted, and errors in the image as well as the reference coordinates cannot be taken into consideration.

The three features discussed above put a bottleneck to the nonlinear least squares estimation model used in obtaining accurate structure and motion. The nonlinear least squares model aims at estimating a vector of parameters $\xi$, from a linearized model $\mathbf{y}=\mathbf{A} \xi+\mathbf{e}$ that includes an observation vector $\mathbf{y}$, a vector of normally distributed errors $\mathbf{e}$, and a matrix of variables A (Felus and Schaffrin 2005). In this model, the underlying assumption is that the design matrix $\mathbf{A}$ is fixed or error-free, which is not often the case in computer vision or photogrammetry since both the image and the reference coordinates may encounter errors. When both the observation vector $\mathbf{y}$ as well as the design matrix A contain errors, the problem is known as error-in-all-variables (EIV). Among the methods put forward to solve an EIV problem is the total least squares (TLS) method (see, e.g., Golub and Van Loan 1980; Felus and Schaffrin 2005; Zwanzig 2006; Neitzel 2010; Grafarend and Awange 2012).

In a recent study, however, Paláncz and Awange (2012) showed that for EIV models, when multiple conflicting objectives exist, or for ill-posed problems (see, e.g., Schaffrin and Snow 2010), the TLS lead to larger global and local residuals and suggested the use of Pareto optimality approach, which 
has been widely used in economics (see, e.g., Hochman and Rodgers 1969; Warr 1982) to estimate the parameters in EIV models. The use of Pareto optimality is necessitated by the fact that many real-world problems involve simultaneous optimization of several incommensurable and often competing objectives (i.e., multi-objectives). Always, there is no single optimal solution, but rather a set of alternative solutions, which are optimal in the wider sense that no other solutions in the search space are superior to them when all objectives are considered (Zitler and Thiele 1999). These solutions, known as Pareto-optimal solutions, were introduced by the Italian economist and sociologist Vilfredo Pareto (1848-1923) (Pressl et al. 2010).

Pareto optimality has been associated with multi-objective problems for quite sometime (see, e.g., Censor 1977; Zitzler and Thiele 1999). Other traditionally available methods for solving multi-objective problems include the goal attainment approach (Wilson and Macleod 1993) and weighted averaging (Coello 1999). Considering the Pareto approach, there occur cases, for example, where the objective to be minimized can be expressed in different forms, resulting in different parametric values for the estimated unknowns at non-zero residiuals. Sometimes these objectives may compete in Pareto sense, namely a small change in the parameters result in an increase of one objective, while decreasing the other. The Pareto optimal set represents a set of optimal solutions between the conflicting objectives, which helps the user to gain a better understanding of the problem structure and supports the decision-maker in choosing the best compromise solution for the considered alternatives. However, in case of lack of such a supervisor, one may select an equilibrium solution from the Pareto-set. 
Examples of the application of Pareto optimality are documented, e.g., by Mirza and Almir (2010) who investigated the application of a multi-objective genetic algorithm based on the Pareto approach as a tool for decision making support in geospatial analysis, and Pressl et al. (2010) who employs Pareto optimality to develop a prototype for a web-based route planning service for people with disabilities who have special requirements on their mobility. Other applications are presented in the works of Lin (1976), Zitler and Thiele (1999), Geisler and Trächtler (2009), Saadatseresht et al. (2009), and Sonnier (2010). In computer vision, the application of pareto optimality is reported e.g., in the works of Dunn et al. (2004) and more recently in Olague and Trujillo $(2011,2012)$.

To help address the bottleneck faced by nonlinear least squares and its improvement, the TLS, the present work proposes the use of Pareto optimality in photogrammetry as a possible solution to the resection-intersection models with EIV. The remeinder of the study is organized as follows: In section2, the photogrammetric resection-intersection problem is formulated leading to a multi-objective EIV model, which is then solved using the Pareto optimality discussed in section 3. Section 4 presents the Han, Manhattan, and Merton architectural examples, while section 5 summarizes the study.

\section{Resection-intersection and the multi-objective problem}

\subsection{Resection-intersection problem}

The fundamental photogrammetric problem amounts to the determination of the interior and exterior orientation parameters of the camera and to obtain the coordinates of the object space of the corresponding points 
measured on the photos (McGlone 1989; Grussenmeyer and Al Khalil 2002).

Photogrammetric resection is the problem of determining the interior and exterior orientation parameters of a camera based on known ground points $\left(X_{j}, Y_{j}, Z_{j}\right)$ and their corresponding photo plane coordinates $\left(x_{j}, y_{j}\right)$. The determination of the orientation parameters is achieved through the geometrical collinearity model equations (e.g., Awange and Kiema 2013)

$$
x_{j}=\eta_{0}-f \frac{r_{11}\left(X_{j}-X_{0}\right)+r_{12}\left(Y_{j}-Y_{0}\right)+r_{13}\left(Z_{j}-Z_{0}\right)}{r_{31}\left(X_{j}-X_{0}\right)+r_{32}\left(Y_{j}-Y_{0}\right)+r_{33}\left(Z_{j}-Z_{0}\right)},
$$

and,

$$
y_{j}=\xi_{0}-f \frac{r_{21}\left(X_{j}-X_{0}\right)+r_{22}\left(Y_{j}-Y_{0}\right)+r_{23}\left(Z_{j}-Z_{0}\right)}{r_{31}\left(X_{j}-X_{0}\right)+r_{32}\left(Y_{j}-Y_{0}\right)+r_{33}\left(Z_{j}-Z_{0}\right)},
$$

where $\eta_{0}, \xi_{0}$ are the coordinates of the perspective center on the photo plane, $f$ is the focal length, $r_{i, j}$ are the elements of the the rotation matrix $\boldsymbol{R}$, and $X_{0}, Y_{0}, Z_{0}$ are the corresponding coordinates of the perspective center in the ground system. The parameters $\eta_{0}, \xi_{0}$ and $f$ are the interior orientation parameters, while the elements of $\boldsymbol{R}$ and $X_{0}, Y_{0}, Z_{0}$ comprise elements of the exterior orientation parameters (e.g., Fig. 1).

\section{Figure 1}

The representation of the mathematical relationship between a point on the photo plane $\left(x_{j}, y_{j}\right)$ and its corresponding point $\left(X_{j}, Y_{j}, Z_{j}\right)$ in the object space can be given without the scaling factor through the collinearity equations (1 and 2). Here, the elements of the rotation matrix are expressed by the elements of the skew matrix $\boldsymbol{S}$ as (Awange et al. 2010) 


$$
\boldsymbol{S}=\left(\begin{array}{ccc}
0 & -c & b \\
c & 0 & -a \\
-b & a & 0
\end{array}\right)
$$

The rotation matrix then becomes (e.g., Awange et al. 2010)

$$
\boldsymbol{R}=\left(\boldsymbol{I}_{3}-\boldsymbol{S}\right)^{-1}\left(\boldsymbol{I}_{3}+\boldsymbol{S}\right)
$$

where $\boldsymbol{I}_{3}$ is a $3 \times 3$ identity matrix. This leads to

$$
\boldsymbol{R}=\left(\begin{array}{ccc}
\frac{1+a^{2}-b^{2}-c^{2}}{1+a^{2}+b^{2}+c^{2}} & \frac{2 a b-2 c}{1+a^{2}+b^{2}+c^{2}} & \frac{2(b+a c)}{1+a^{2}+b^{2}+c^{2}} \\
\frac{2(a b+c)}{1+a^{2}+b^{2}+c^{2}} & \frac{1-a^{2}+b^{2}-c^{2}}{1+a^{2}+b^{2}+c^{2}} & -\frac{2(a-b c)}{1+a^{2}+b^{2}+c^{2}} \\
\frac{2(-b+a c)}{1+a^{2}+b^{2}+c^{2}} & \frac{2(a+b c)}{1+a^{2}+b^{2}+c^{2}} & \frac{1-a^{2}-b^{2}+c^{2}}{1+a^{2}+b^{2}+c^{2}}
\end{array}\right) .
$$

In a general case, there are 9 parameters to be computed, namely, the interior orientation parameters $\left(\eta_{0}, \xi_{0}\right.$ and $\left.f\right)$, as well as the exterior orientation parameters $\left(a, b, c\right.$ and $\left.X_{0}, Y_{0}, Z_{0}\right)$. Every corresponding point-pair provides 2 collinearity equations, therefore to compute the 3 internal and the 6 external parameters, one needs a minimum 5 corresponding point-pairs. Consequently, even in the minimum case, we have an overdetermined system $(5 \times 2=10$ equations and 9 unknowns). In practice, there are more measured points than the minimum leading to an overdetermined system of equations, which can be solved for the parameters in a least squares sense (i.e., resection).

In its implicit form, the collinearity equations (1 and 2) can be written as

$$
\begin{gathered}
\rho_{x_{j}}=\left(x_{j}-\eta_{0}\right)\left(r_{31}\left(X_{j}-X_{0}\right)+r_{32}\left(Y_{j}-Y_{0}\right)+r_{33}\left(Z_{j}-Z_{0}\right)\right)+ \\
f r_{11}\left(X_{j}-X_{0}\right)+r_{12}\left(Y_{j}-Y_{0}\right)+r_{13}\left(Z_{j}-Z_{0}\right)=0 .
\end{gathered}
$$


204

and

$$
\begin{gathered}
\rho_{y_{j}}=\left(y_{j}-\xi_{0}\right)\left(r_{31}\left(X_{j}-X_{0}\right)+r_{32}\left(Y_{j}-Y_{0}\right)+r_{33}\left(Z_{j}-Z_{0}\right)\right)+ \\
f r_{21}\left(X_{j}-X_{0}\right)+r_{22}\left(Y_{j}-Y_{0}\right)+r_{23}\left(Z_{j}-Z_{0}\right)=0,
\end{gathered}
$$

where the elements $r_{i, j}$ 's of the rotation matrix $R$ depend on the elements $(a, b, c)$ of the skew matrix $S$. Considering $n$ points on a photo-plane, one has $2 n$ equations to estimate the parameter $\pi=\left(a, b, c, X_{0}, Y_{0}, Z_{0}, \eta_{0}, \xi_{0}\right.$, f) belonging to this image.

In real situations, when the initial values for the parameters above are not known, the global solution of the overdetermined polynomial equations (4) and (5) is not trivial. One possibility is to solve a determined subsystem with numerical Groebner basis or alternatively with linear homotopy method, then employ the results as initial values for the extended Newton method for solving the overdetermined system (see e.g., Awange et al. 2010). Undoubtedly, the most effective global method is the global minimization methods. Here we use random - search method to minimize the residual of the equations in a least square sense. The objective function based on the implicit equations (4 and 5$)$ is

$$
G_{I}(\pi)=\sum_{j=1}^{n}\left(\rho_{x_{j}}^{2}+\rho_{y_{j}}^{2}\right)
$$

Frequently, the same camera is used to aquire the two photo-planes. Therefore the determined internal orientation parameters $\left(f, \eta_{0}, \xi_{0}\right)$ computed from the data of the two photo-planes should be the same. However the simultaneously estimated parameters from both photo-planes requires the solution of an ill-conditioned problem. Therefore the parameters are mostly estimated independently for the two photo-planes accepting that $f^{(1)} \approx f^{(2)}, \eta_{0}^{(1)} \approx \eta_{0}^{(2)}$, 
and $\xi_{0}^{(1)} \approx \xi_{0}^{(2)}$. Once the interior and exterior orientation parameters of the two cameras have been determined through resection, the next step entails the determination of the position $(X, Y, Z)$ of a point in $3 \mathrm{D}$ space from at least 2 photo image coordinates $(x, y)$ and $(u, v)$ registered on (at least) two photo planes (e.g., Fig. 2) through the procedure known as intersection.

\section{Figure 2}

To determine each ground coordinate $\left(X_{j}, Y_{j}, Z_{j}\right)$, the corresponding coordinates on the two photo planes $\left(x_{j}, y_{j}\right)$, and $\left(u_{j}, v_{j}\right)$ are needed. It means that to compute the space (ground) coordinates of a point, we have 4 collinearity equations ( 2 equations belonging to each photo-plane) being linear in the 3 unknowns $(X, Y, Z)$. Therefore, theoretically, any 3 equations could be considered although it is more reasonable to carry out the computation simultaneously as a linear regression problem.

The two collinearity equations for the first photo plane are

$$
\begin{gathered}
f^{(1)}\left(\left(X-X_{01}\right) r_{1,1}+\left(Y-Y_{01}\right) r_{1,2}+\left(Z-Z_{01}\right) r_{1,3}\right)+ \\
\left(x-\eta_{01}\right)\left(\left(X-X_{01}\right) r_{3,1}+\left(Y-Y_{01}\right) r_{3,2}+\left(Z-Z_{01}\right) r_{3,3}\right)=0
\end{gathered}
$$

and,

$$
\begin{gathered}
f^{(1)}\left(\left(X-X_{01}\right) r_{2,1}+\left(Y-Y_{01}\right) r_{2,2}+\left(Z-Z_{01}\right) r_{2,3}\right) \\
+\left(y-\xi_{01}\right)\left(\left(X-X_{01}\right) r_{3,1}+\left(Y-Y_{01}\right) r_{3,2}+\left(Z-Z_{01}\right) r_{3,3}\right)=0 .
\end{gathered}
$$

239 Similarly the equations for the second photo plane are

$$
\begin{gathered}
f^{(2)}\left(\left(X-X_{02}\right) R_{1,1}+\left(Y-Y_{02}\right) R_{1,2}+\left(Z-Z_{02}\right) R_{1,3}\right) \\
+\left(u-\eta_{02}\right)\left(\left(X-X_{02}\right) R_{3,1}+\left(Y-Y_{02}\right) R_{3,2}+\left(Z-Z_{02}\right) R_{3,3}\right)=0
\end{gathered}
$$


240 and,

$$
\begin{gathered}
f^{(2)}\left(\left(X-X_{02}\right) R_{2,1}+\left(Y-Y_{02}\right) R_{2,2}+\left(Z-Z_{02}\right) R_{2,3}\right) \\
+\left(v-\xi_{02}\right)\left(\left(X-X_{02}\right) R_{3,1}+\left(Y-Y_{02}\right) R_{3,2}+\left(Z-Z_{02}\right) R_{3,3}\right)=0 .
\end{gathered}
$$

\subsection{Resection-intersection objectives}

Traditionally, the system of the collinearity equations employed to estimate the parameters of the $i$-th camera $\left(\pi_{i}\right)$ can be written in implicit form,

$$
P_{j}\left(\pi_{i}, X_{j}, Y_{j}, Z_{j}, x_{j}, y_{j}\right)=0, j=1, \ldots, n \geq 5
$$

where $\left(X_{j}, Y_{j}, Z_{j}\right)$ and $\left(x_{j}, y_{j}\right)$ are the measured coordinates corresponding to the ground and photo plane systems, respectively. Having a minimum of two cameras with known parameters, the coordinates of an optional object point $X, Y, Z$ can be computed from the coordinates of two projected points in two separate images $(x, y)$ and $(u, v)$ employing 4 collinearity equations

$$
\begin{aligned}
& x=p_{x}\left(\pi_{1}, X, Y, Z\right) \\
& y=p_{y}\left(\pi_{1}, X, Y, Z\right) \\
& u=p_{u}\left(\pi_{2}, X, Y, Z\right) \\
& v=p_{v}\left(\pi_{2}, X, Y, Z\right),
\end{aligned}
$$

or in implicit form,

$$
\begin{aligned}
& P_{x}\left(\pi_{1}, X, Y, Z, x, y\right)=0 \\
& P_{y}\left(\pi_{1}, X, Y, Z, x, y\right)=0 \\
& P_{u}\left(\pi_{2}, X, Y, Z, u, v\right)=0 \\
& P_{v}\left(\pi_{2}, X, Y, Z, u, v\right)=0 .
\end{aligned}
$$

The problem is overdetermined with 4 equations and 3 unknowns, and the least squares method can be used again (one-point intersection in Fig. 2). 
In order to formulate an explicit multi-objective EIV-model, the one-point intersection problem in Eqn. (12) is expressed in a least squares sense employing symbolic pseudo-inverse. The coordinates of an object point $(X, Y, Z)$ is expressed explicitly as the functions of the corresponding photo plane coordinates $(x, y)$ and $(u, v)$ as

$$
\begin{aligned}
& X=p_{X}\left(\pi_{1}, \pi_{2}, x, y, u, v\right) \\
& Y=p_{Y}\left(\pi_{1}, \pi_{2}, x, y, u, v\right) \\
& Z=p_{Z}\left(\pi_{1}, \pi_{2}, x, y, u, v\right)
\end{aligned}
$$

from which the unknown camera parameters $\left(\pi_{1}, \pi_{2}\right)$ are determined from the explicit objective function

$$
\begin{gathered}
G_{\mathrm{XYZ}}\left(\pi_{1}, \pi_{2}\right)=\sum_{j=1}^{n} W_{X_{j}}\left(X_{j}-p_{X}\left(\pi_{1}, \pi_{2}, x_{j}, y_{j}, u_{j}, v_{j}\right)\right)^{2}+ \\
W_{Y_{j}}\left(Y_{j}-p_{Y}\left(\pi_{1}, \pi_{2}, x_{j}, y_{j}, u_{j}, v_{j}\right)\right)^{2}+ \\
W_{Z_{j}}\left(Z_{j}-p_{Z}\left(\pi_{1}, \pi_{2}, x_{j}, y_{j}, u_{j}, v_{j}\right)\right)^{2}
\end{gathered}
$$

which is constructed using every weighted $j^{\text {th }}$ ground point $\left(X_{j}, Y_{j}, Z_{j}\right)$ and their corresponding photo planes coordinates $\left(x_{j}, y_{j}\right)$ and $\left(u_{j}, v_{j}\right), j=1, \ldots, n$. Now this objective function has a clear physical interpretation, namely, it is the sum of the square of the differences between the measured and the computed ground coordinates. Its minimization results into the orientation parameters of both cameras simultaneously (i.e., resection). In order to estimate the parameters $\pi_{1}, \pi_{2}$, a different objective function can be determined on the basis of the weighted measured and computed coordinates of the photo 
plane points as

$$
\begin{gathered}
G_{\text {xyuv }}\left(\pi_{1}, \pi_{2}\right)=\sum_{j=1}^{n} w_{x_{j}}\left(x_{j}-p_{x}\left(\pi_{1}, X_{j}, Y_{j}, Z_{j}\right)\right)^{2}+ \\
w_{y_{j}}\left(y_{j}-p_{y}\left(\pi_{1}, X_{j}, Y_{j}, Z_{j}\right)\right)^{2}+ \\
w_{u_{j}}\left(u_{j}-p_{u}\left(\pi_{2}, X_{j}, Y_{j}, Z_{j}\right)\right)^{2}+ \\
w_{v_{j}}\left(v_{j}-p_{v}\left(\pi_{2}, X_{j}, Y_{j}, Z_{j}\right)\right)^{2}
\end{gathered}
$$

Since there exists two competing objectives (Eqns. 15 and 16), probably the best strategy is to find a trade-off between them, namely, to consider their linear combinations resulting from a mono-objective function

$$
G\left(\pi_{1}, \pi_{2}, \lambda\right)=\lambda G_{\mathrm{XYZ}}\left(\pi_{1}, \pi_{2}\right)+(1-\lambda) G_{\mathrm{xyuv}}\left(\pi_{1}, \pi_{2}\right),
$$

where $\lambda$ are the weighting parameters. This is a classical multi-objective optimization (MO) problem, where the objectives $G_{\mathrm{XYZ}}$ and $G_{\mathrm{xyuv}}$ are competing with no unique solution. Instead, the concept of non-inferiority (also called Pareto optimality) must be used to characterize the objectives (e.g., Censor 1977). The solution of a MO problem is not a particular value, but a set of values of the decision variables (called Perato-set). For each element in this set, none of the objective functions can be increased without a decrease of some of the remaining objective functions. Every such a decision-value is referred to as Pareto-optimal.

Since the dimensions of the different objectives are different, in our case, the ground coordinates are in m-units and the image coordinates in pixel, it is reasonable to introduce normalized, dimensionless multi-objective functions, for example, Eqns. (15) and (16) can be written as

$$
\tilde{G}_{\mathrm{XYZ}}\left(\pi_{1}, \pi_{2}\right)=\frac{G_{\mathrm{XYZ}}\left(\pi_{1}, \pi_{2}\right)-G_{\mathrm{XYZmin}}}{G_{\mathrm{XYZmax}}-G_{\mathrm{XYZmin}}},
$$


285

and

$$
\tilde{G}_{\text {xyuv }}=\frac{G_{\text {xyuv }}-G_{\text {xyuvmin }}}{G_{\text {xyuvmax }}-G_{\text {xyuvmin }}} .
$$

The dimensionless form of the mono-objective function then becomes

$$
\tilde{G}\left(\pi_{1}, \pi_{2}, \lambda_{1}, \lambda_{2}\right)=\lambda \tilde{G}_{\mathrm{XYZ}}\left(\pi_{1}, \pi_{2}\right)+(1-\lambda) \tilde{G}_{\mathrm{xyuv}}\left(\pi_{1}, \pi_{2}\right)
$$

\subsection{An alternative development of the multi - objective problem}

The symbolic form of the explicit expression of the collinearity equations for the space coordinates $(\mathrm{X}, \mathrm{Y}, \mathrm{Z})$ with one-point intersection is possible if there are only two photo-planes. In that case, Eq. (12) or Eq. (13) can be solved for space coordinates as an overdetermined linear system using symbolic pseudoinverse. To get an alternative form of Eq. (15) for three or more photo-planes, which does not require the explicit form Eq. (14), let us introduce the adjustments of the space coordinates $\Delta \mathrm{X}_{j}, \Delta \mathrm{Y}_{j}, \Delta \mathrm{Z}_{j}$. Then, Eq. (13) can be written for the i-th camera (photo-plane) as

$$
\begin{aligned}
& P_{X}\left(\pi_{i}, X_{j}+\Delta \mathrm{X}_{j}, Y_{j}+\Delta \mathrm{Y}_{j}, Z_{j}+\Delta \mathrm{Z}_{j}, x_{j}{ }^{(i)}, y_{j}^{(i)}\right)=0, j=1, \ldots, n \\
& P_{Y}\left(\pi_{i}, X_{j}+\Delta \mathrm{X}_{j}, Y_{j}+\Delta \mathrm{Y}_{j}, Z_{j}+\Delta \mathrm{Z}_{j}, x_{j}{ }^{(i)}, y_{j}{ }^{(i)}\right)=0, j=1, \ldots, n
\end{aligned}
$$

where $i=1,2, \ldots, m$ is the number of the photo-planes. Now the objective function $G_{\mathrm{XYZ}}\left(\pi_{1}, \pi_{2}, \ldots \pi_{n}\right)$ can be written as

$$
G_{\mathrm{XYZ}}\left(\pi_{1}, \pi_{2}, \ldots, \pi_{m}\right)=\sum_{j=1}^{n} W_{X_{j}} \Delta \mathrm{X}_{j}{ }^{2}+W_{Y_{j}} \Delta \mathrm{Y}_{j}{ }^{2}+W_{Z_{j}} \Delta \mathrm{Z}_{j}{ }^{2}
$$

with Eq. (19) as a constraint. The payment for avoiding the explicit expression of the space coordinates is relatively high. Using the explicit form of $(X, Y, Z)$, we need to compute $9 m$ unknown parameters. However, the number of the unknowns parameters will be $9 m+3 n$ in case Eq. (20) is used. 
In addition, one should solve an optimization problem under constrains. For example, in case of two photo-planes $(\mathrm{m}=2)$ with $\mathrm{n}=5$ points on each, there are $9 \times 2=18$ unknown parameters versus $18+3 \times 5=33$.

\section{Pareto optimality}

\subsection{Basic definitions}

In many real - life situations, there are multi-objective optimality problems, which means that there are more than one objective to be minimized or maximized. In cases where all of the objective functions either increase or decrease, there exists no optimum. However, in regions where these objective functions are competing or conflicting with each other, meaning that a small change in the independent variables will result not only in an increase of one objective function, but also a decrease in the others, an optimum can exist. We call such regions feasible regions for optimal solutions.

A solution in such a region is said to be a Pareto optimal solution if it is not dominated by any other solution in that region. Pareto Optimality is defined as follows (Marler and Arora 2004):

Definition: A point, $\mathbf{x}^{*} \in \mathbf{X}$, is Pareto optimal iff there does not exist another point, $\mathbf{x} \in \mathbf{X}$, such that $\mathbf{F}(\mathbf{x}) \leq \mathbf{F}\left(\mathbf{x}^{*}\right)$, and $\mathbf{F}_{i}(\mathbf{x})<\mathbf{F}_{i}\left(\mathbf{x}^{*}\right)$ for at least one function.

From the definition above, the Pareto optimal solution is therefore a set of solutions, rather than a single one. The independent variables representing these solutions in the variable space form a Pareto-set, and the corresponding values of the objective functions are labeled as the Pareto-front. In our case (e.g., Eqn. 15), the objective functions are convex, therefore the Pareto-front 
is also convex and connected.

The selection of a single optimum solution from the Pareto-set needs a trade-off strategy to be implemented by the user (decision maker). The Pareto balanced solution as a single solution minimizes the sum of the values of the dimensionless objective functions belonging to the Pareto-front. This optimal solution is balanced (neutral), which means it has a preference for none of the objective functions. For more details on the Pareto optimality approach, we refer the reader to Marler and Arora (2004) and Paláncz and Awange (2012).

\subsection{The multi-objective optimization of the resection-intersection problem}

In order to determine the normalized dimensionless objective in Eqn. (18), the individual minimum and maximum values of the explicit objectives in Eqs. (15) and (16) are computed via a local method (LevenbergMarquardt) with the results of the traditional solutions as initial guess values. The maximum values are then computed by substituting the individual minimums into the counterpart objectives. The multi-objective problem is then converted into a mono-objective problem by introduction the normalized, dimensionless objective function (e.g., Eqn. (18)).

\section{Pareto application to photogrammetric resection-intersection prob- lem}

The traditional methods mostly based on implicit equations prefer to minimize the residual of these equations and result in much better fitting in the image coordinates than in the space coordinates (see the Manhattan example in Sect. 4.2). However, one may need a balance between these two 
types of errors (camera calibration) or may prefer to minimize the error in the space coordinates (see the architectural reconstruction example in Sect. 4.3). The suggested method based on Pareto optimum can provide a flexible technique to achieve the minimization of the selected objective of the user in a properly controlled way.

To demonstrate the capability of the suggested method, three examples are presented. The first example in Sect. 4.1 is adopted from the literature, which is a real outdoor experiment estimating the orientation parameters of a camera from two close range images acquired by a nonmetric digital camera. We used this example to compare the results of our algorithm with those computed using the traditional approach, as well as to check the robustness of our algorithm in estimating all camera parameters simultaneously. The second example in Sect. 4.2 is a camera calibration problem, where the interior and exterior orientation parameters are estimated on the basis of the collinearity equations, employing the data of a Manhattan-type test field. In this example, the reduction of the transformation errors on the image, as well as on the space coordinates are equally important. The third example in Sect. 4.3 considers an architectural reconstruction problem, where real field data of a Merton college court in Oxford (UK) is applied to estimate the camera matrices. In this case, in order to reconstruct the building space coordinates from the image coordinates, one has to reduce the errors in the space coordinates.

First, the traditional parameter estimation is presented, using implicit form of the corresponding equations. Then, employing numerical intersection via linear least squares (LLS), the quality of the traditional approach is 
evaluated on the basis of the corresponding measured values of the ground and the image coordinates.

Next, the one-point intersection problem is solved in a symbolic form by computing the symbolic pseudo-inverse of the linear overdetermined system to give explicit expressions for the space coordinates (e.g., Eqn. 14). To determine the unknown parameters, the two competing objective functions for sum of squares of the coordinate errors (e.g., Eqns. 15 and 16) are formulated. As a last step, the Pareto-front is computed and a single element of the Pareto-front selected as the Pareto optimal solution, which provides the smallest global error for the image as well as for the space coordinates, separately. In addition the quality of the suggested method is assessed by considering both the global and local errors, and comparing them to those of the traditional method.

\subsection{Step by step solution of the Han's Example}

This example is based on the problem adopted from Han et al. (2011).

Han for our disposal. During this outdoor experiment, close-range images from two exposure stations were acquired using a Nikon D-80 nonmetric digital single-lense reflex (DSLR) camera, see Fig. 3.

\section{Figure 3}

The image resolution was $2896 \times 1944$ pixels, with pixel size of about 0.8 $\mathrm{cm}$ for a target that is $20 \mathrm{~m}$ away from the camera. The same test was also performed using distorted camera positions by manually adding $30 \mathrm{~cm}$ errors to its accurate positions. The coordinates for the check and control points as well as the two camera stations were surveyed and precisely determined by a 
total station. They used pre-computed parameters $f, \eta_{0}, \xi_{0}, a, b, c, X_{0}, Y_{0}, Z_{0}$ for both images and estimated the elements of the rotation matrix (the rotation angles) from the measurements for both images separately.

In our computation all of the interior and exterior parameters were computed simultaneously for both images from the measurements, where the interior orientation parameters were allowed to take different values for different images. In this way, we could check the consistency of the result of our parameter estimation. Employing our algorithm (described in details in the Appendix), the Pareto-set and the Pareto-front were computed for these parameters, and the Pareto balanced solution - the solution representing the very point of the Pareto front which is closest to the ideal point in $L_{1}$ norm was selected. Table 1 shows the values of the conflicting objective functions in case of the two extreme solutions $(\lambda=0$ and $\lambda=1)$, as well as in case of the Pareto optimal solution $(\lambda=0.34)$ where the $\tilde{G}$ has its minimum.

\section{Table 1}

Table 2 represents the camera parameters corresponding to the Pareto optimum solution as well as the results of Han et al. (2011) for both images. It can be seen, that although we compute all parameters from the measured data simultaneously- which is a difficult computation since the problem is an ill conditioned one - we got close results to those of Han et al. (2011) who estimated the interior and exterior parameters parameters separately. This indicates the robustness of the suggested Pareto algorithm. In addition the RMSE of the space coordinates in our case was $0.024 \mathrm{~m}$ while Han et al. (2011) reported a value of $0.028 \mathrm{~m}$. This study illustrated again that employing Pareto-optimality, one can decide which error is important to reduce the 
435

RMSE of $G_{\mathrm{XYZ}}$ (space coordinate side) or the RMSE of $G_{\text {xyuv }}$ (photo plane coordinate side) when estimate the parameters.

\section{Table 2}

\subsection{The Manhattan-type test example}

\subsubsection{Traditional solution}

First, the traditional solution of the resection problem is applied to the data in Table 3 from the Manhattan-type test field in Fig. (4) (Fekete and Schrott 2008). Let us consider the first 9 points as training and the last 6 points as validation points. The parameter estimation is then carried out for the training points via solving nonlinear least squares problem represented by $9 \times 4$ implicit collinearity equations. The validation points serves as a check for the quality of the proposed procedure. The points were labeled in a way that the region of training points covered the validation points. Figure 5 shows the Voronoi-cells of the training and the validation points. The validation points are numbered as $1-9$, and the training points are numbered 10 - 15 for both photo planes.

\section{Table 3}

\section{Figure 5}

The results of the computation are presented in Table 4 . The correspond- 
437

ing rotation matrices are

$$
\begin{gathered}
\boldsymbol{R}_{1}=\left(\begin{array}{ccc}
0.98586 & -0.0174065 & 0.166662 \\
0.0403779 & 0.98996 & -0.135455 \\
-0.162631 & 0.140269 & 0.976666
\end{array}\right), \\
\boldsymbol{R}_{2}=\left(\begin{array}{ccc}
0.995006 & -0.0257385 & -0.0964346 \\
-0.0140006 & 0.920634 & -0.390176 \\
0.0988235 & 0.389577 & 0.915676
\end{array}\right) .
\end{gathered}
$$

Substituting these parameters into the collinearity equations, the errors in the image coordinates $(\Delta \mathrm{x}, \Delta \mathrm{y})$ as the difference of the measured and computed values are determined. Table 5 shows these errors as well as those of the $L_{2}$-norm of the error vectors, $\left(\Delta \mathrm{x}_{i}, \Delta \mathrm{y}_{i}\right)^{T}$ for both photo-planes. Since there are 15 points on each photo plane, our linear system consists of 60 linear equations containing 45 unknowns. Substituting the parameters computed from the resection into the collinearity equations and solving the linear least squares intersection problem, the space coordinates are obtained. The differences between the measured and computed values $(\Delta \mathrm{X}, \Delta \mathrm{Y}, \Delta \mathrm{Z})$, as well as the $L_{2}$-norm of the error vectors $\left(\Delta \mathrm{X}_{i}, \Delta \mathrm{Y}_{i}, \Delta \mathrm{Z}_{i}\right)^{T}$ are presented in Table 6 .

\section{Table 5}

Table 6

\subsubsection{The suggested Pareto optimality method}

In order to improve this algorithm, two features can be considered (i) the interior and exterior parameters are estimated simultaneously for both 
photo planes using resection (bundle adjustment), or (ii) the parameters are determined by solving the multi-objective optimization problem using Pareto optimality with the objectives $G_{\mathrm{xyuv}}$ and $G_{\mathrm{XYZ}}$ constructed from the explicit expressions of the images as well as from the ground coordinates (e.g., Eqs. 15 and 16). This last feature represents the real novelty of our contribution.

In order to get this explicit expression for the space coordinates in $G_{\mathrm{XYZ}}$, the one-point intersection problem is solved using Mathematica computer algebra system. After the computation of the dimensionless form of the conflicting objective functions, the mono-objective function $\tilde{G}$ in Eqn. (18) will be minimized with the parameters $\lambda \in[0,1]$ leading to the Pareto-set. As an illustration, Fig. 6 shows the parameter $a$, one of the element of the skew matrix $\mathbf{S}$ as function of $\lambda$. Using Levenberg-Marquardt method in parallel way on i7 Intel Nehalem processor with 4 cores (8 threads), the computational speed-up was 1.89 seconds (i.e., about 2 times faster than a single core machine) in the case of the two photo-planes.

\section{Figure 6}

The Pareto-front, i.e., the corresponding values of the dimensionless objective functions to the Pareto-set, together with the Pareto balanced solution belonging to $\lambda=0.5$ as well as the result of the traditional solution based on the implicit equations are shown in Fig. 7.

\section{Figure 7}

Figure (7) shows that the traditional solution using implicit form of the collinearity equations is not Pareto optimal, since it does not belong to the 
Pareto-front. It is remarkable that the left-hand side of the Pareto-front in the figure is very steep, where the minimum of residual of the photo-plane coordinates $\tilde{G}_{\text {xyuv }}$ is changing rapidly, while there is practically no change in the minimum of the residual of the ground coordinates, $\tilde{G}_{\mathrm{XYZ}}$ on the righthand side of the figure.

\subsubsection{Computation of the selected single solution}

Although the Pareto balanced optimum belonging to $\lambda=0.5$ provides a minimum for the normalized total objective (mono-objective), $\tilde{G}_{\mathrm{XYZ}}+\tilde{G}_{\text {xyuv }}$ $=0.0579521$ in Eqn. (18), which is considerably smaller than that of the traditional solution (1.21389), namely it has considerably smaller residual for the ground coordinates $\tilde{G}_{\mathrm{XYZ}}=0.0178549$ than the traditional solution (1.21375), its residual for the photo-plane coordinates however is greater $\tilde{G}_{\text {xyuv }}=0.0400972$ than that of the traditional solution $(0.00014471)$. Fortunately, there exists a portion of the Pareto-front, under the horizontal line, where the optimums represent a superior region over the traditional solution, i.e., where both normalized objectives are smaller than those of the traditional solutions (see Fig. 8).

\section{Figure 8}

This section belongs to the parameter values of $\lambda \leq 0.00137153$. Let us select from this section the optimal solution which belongs to $\lambda=0.00137$. The corresponding Pareto optimal transformations parameters are shown in Table 7. Now, this selected single solution provides smaller residuals (global errors) for both objectives than the traditional solution as indicated in Table 8. The mean and variance of the local error vectors are presented in case of 
the traditional and the Pareto optimum solution in Table 9. As is expected, according to the global result, the selected single optimum solution has reduced the error in the space coordinates considerably, without practical error increasing in the image coordinates. The reason for this is due to the fact that the traditional solution has a strong preference to minimize the image coordinates instead of errors of the space coordinates (see Fig. 7).

Table 7

Table 8

Table 9

\subsection{Architectural reconstruction problem}

There has been intensive effort in Photogrammetry and Computer Vision research on reconstruction of architecture from photographs. In the following example, the Pareto optimality approach is employed for reconstruction of a Merton College court in Oxford. The data is adopted from Werner at al. (1999) and are presented in Fig. 9 as well as in Table 10. The points in 3D can be seen in Fig. 10.

Figure 9

Figure 10

Most frequently, in such photogrammetric applications, instead of collinearity equations, the relation between the coordinates of points in 3D space and the corresponding coordinates on an image can be represented by the camera 
516

matrix $\boldsymbol{C}$ given as

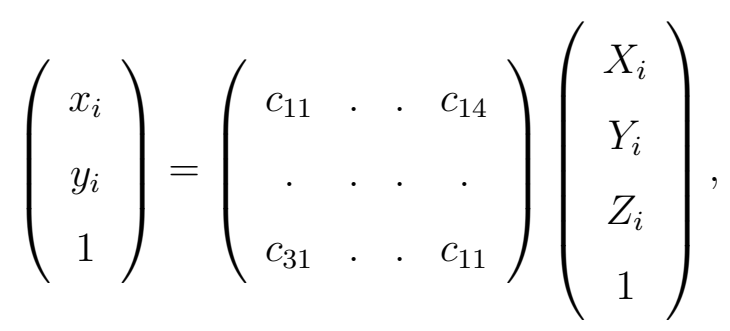

and

and

Table 10

\subsubsection{Traditional computation of the camera matrix}

First, using these data the estimation of the elements of the two camera matrices will be carried out employing implicit equations derived from these matrices. The explicit equations for the image coordinates are,

$$
x_{i}-\frac{\left(c_{11}, c_{12}, c_{13}, c_{14}\right) \cdot\left(X_{i}, Y_{i}, Z_{i}, 1\right)^{T}}{\left(c_{31}, c_{32}, c_{33}, c_{34}\right) \cdot\left(X_{i}, Y_{i}, Z_{i}, 1\right)^{T}}=0
$$

$$
y_{i}-\frac{\left(c_{21}, c_{22}, c_{23}, c_{24}\right) \cdot\left(X_{i}, Y_{i}, Z_{i}, 1\right)^{T}}{\left(c_{31}, c_{32}, c_{33}, c_{34}\right) \cdot\left(X_{i}, Y_{i}, Z_{i}, 1\right)^{T}}=0,
$$

with their implicit forms given as

$$
-c_{14}+c_{34} x_{i}-c_{11} X_{i}+x_{i} X_{i}-c_{12} Y_{i}+c_{32} x_{i} Y_{i}-c_{13} Z_{i}+c_{33} x_{i} Z_{i}=0
$$

$$
-c_{24}-c_{21} X_{i}+c_{34} y_{i}+X_{i} y_{i}-c_{22} Y_{i}+c_{32} y_{i} Y_{i}-c_{23} Z_{i}+c_{33} y_{i} Z_{i}=0 .
$$


537

539

We have 25 points, therefore to compute the elements of the two camera matrices, there are 50 equations for each camera. This is a linear regression problem. The resulting camera matrices are

$$
C_{1}=\left(\begin{array}{crrr}
549.624 & -4237.12 & 1778.75 & 39094.4 \\
-3970.36 & -1084.98 & -1206.85 & 38254.2 \\
1 & -2.60846 & -2.64161 & 77.6154
\end{array}\right)
$$

and

$$
C_{2}=\left(\begin{array}{cccc}
640.323 & -1684.9 & 789.539 & 13121 . \\
-1595.68 & -285.016 & -481.946 & 15709.3 \\
1 & -0.390185 & -0.809379 & 25.7232
\end{array}\right) .
$$

Now, let us employ the Pareto optimum solution. As its first step, we should solve the one-point intersection problem.

\subsubsection{Symbolic solution of one-point intersection problem}

In this case, the four equations are

$$
\begin{aligned}
& -c_{14}+c_{34} x-c_{11} X+x X-c_{12} Y+c_{32} x Y-c_{13} Z+c_{33} x Z_{i}=0 \\
& -c_{24}-c_{21} X+c_{34} y+X y-c_{22} Y+c_{32} y Y-c_{23} Z+c_{33} y Z=0
\end{aligned}
$$

for the first image and

$$
\begin{aligned}
& -X c_{11}-Y c_{12}-Z c_{13}-c_{14}+X u+Y c_{32} u+Z c_{33} u+c_{34} u \\
& -X c_{21}-Y c_{22}-Z c_{23}-c_{24}+X v+Y c_{32} v+Z_{i} c_{33} v+c_{34} v
\end{aligned}
$$

for the second image. The symbolic solution of this overdetermined system for $X, Y, Z$ is then computed using Mathematica computer algebra system. For $X$ for example, we have

$$
p_{X}\left(\pi_{1}, \pi_{2}, x_{j}, y_{j}, u_{j}, v_{j}\right)=X
$$


where the parameters are the elements of the camera matrices, namely

$$
\pi_{1}=\left(c_{11}, c_{12}, c_{13}, c_{14}, c_{21}, c_{22}, c_{23}, c_{24}, c_{32}, c_{33}\right),
$$

and

$$
\pi_{2}=\left(\mathbf{c}_{11}, \mathbf{c}_{12}, \mathbf{c}_{13}, \mathbf{c}_{14}, \mathbf{c}_{21}, \mathbf{c}_{22}, \mathbf{c}_{23}, \mathbf{c}_{24}, \mathbf{c}_{32}, \mathbf{c}_{33}\right)
$$

\section{Multiobjective optimization problem}

Now, the competing objective functions based on the explicit equations can be defined for the image coordinates as

$$
G_{\mathrm{xy}}\left(\pi_{1}\right)=\sum_{i=1}^{n}\left[\left(x_{i}-\frac{\left(c_{11}, c_{12}, c_{13}, c_{14}\right) \cdot\left(X_{i}, Y_{i}, Z_{i}, 1\right)^{T}}{\left(c_{31}, c_{32}, c_{33}, c_{34}\right) \cdot\left(X_{i}, Y_{i}, Z_{i}, 1\right)^{T}}\right)^{2}+\left(y_{i}-\frac{\left(c_{21}, c_{22}, c_{23}, c_{24}\right) \cdot\left(X_{i}, Y_{i}, Z_{i}, 1\right)^{T}}{\left(c_{32}, c_{32}, c_{33}, c_{34}\right) \cdot\left(X_{i}, Y_{i}, Z_{i}, 1\right)^{T}}\right)^{2}\right]
$$

and

$$
G_{\mathrm{uv}}\left(\pi_{2}\right)=\sum_{i=1}^{n}\left[\left(u_{i}-\frac{\left(\mathbf{c}_{11}, \mathbf{c}_{12}, \mathbf{c}_{13}, \mathbf{c}_{14}\right) \cdot\left(X_{i}, Y_{i}, Z_{i}, 1\right)^{T}}{\left(\mathbf{c}_{31}, \mathbf{c}_{32}, \mathbf{c}_{33}, \mathbf{c}_{34}\right) \cdot\left(X_{i}, Y_{i}, Z_{i}, 1\right)^{T}}\right)^{2}+\left(v_{i}-\frac{\left(\mathbf{c}_{21}, \mathbf{c}_{22}, \mathbf{c}_{23}, \mathbf{c}_{24}\right) \cdot\left(X_{i}, Y_{i}, Z_{i}, 1\right)^{T}}{\left(\mathbf{c}_{31}, \mathbf{c}_{32}, \mathbf{c}_{33}, \mathbf{c}_{34}\right) \cdot\left(X_{i}, Y_{i}, Z_{i}, 1\right)^{T}}\right)^{2}\right],
$$

then

$$
G_{\text {xyuv }}\left(\pi_{1}, \pi_{2}\right)=G_{\mathrm{xy}}\left(\pi_{1}\right)+G_{\mathrm{uv}}\left(\pi_{2}\right)
$$

For the space coordinates,

$$
\begin{gathered}
G_{\mathrm{XYZ}}\left(\pi_{1}, \pi_{2}\right)=\sum_{i=j}^{n}\left[\left(X_{j}-p_{X}\left(\pi_{1}, \pi_{2}, x_{j}, y_{j}, u_{j}, v_{j}\right)\right)^{2}+\left(Y_{j}-p_{Y}\left(\pi_{1}, \pi_{2}, x_{j}, y_{j}, u_{j}, v_{j}\right)\right)^{2}\right]+ \\
{\left[\left(Z_{j}-p_{Z}\left(\pi_{1}, \pi_{2}, x_{j}, y_{j}, u_{j}, v_{j}\right)\right)^{2}\right] .}
\end{gathered}
$$

The corresponding mono-objective problem leads to Eqn. (18). The Paretoset is computed as before, and the corresponding Pareto-front with the Pareto balanced solution presented in Fig. 11. Since our aim is a 3D reconstruction, we prefer to reduce the objective $G_{\mathrm{XYZ}}$, i.e., the errors in the space coordinates. The selected optimum should be on the Pareto-front, where considerable reduction only in $G_{\mathrm{XYZ}}$ is not possible since this would increase 
557 ${ }_{567}[\Delta X, \Delta Y, \Delta Z]^{T}$.

568 point in Fig. 11.

and

$G_{\mathrm{xyuv}}$. Therefore the optimum was selected at $\lambda=0.1$ as shown by the green

\section{Figure 11}

Table 11 shows the global errors of the different solutions. It can be seen that in our case, the implicit solution reduces the error of the images coordinates efficiently, but results in a bigger error in the space coordinates. The corresponding camera matrices are

$$
C_{1}=\left(\begin{array}{cccc}
215.792 & -16133.623 & 4996.427 & 156824.915 \\
-15154.350 & -4002.361 & -4629.336 & 144267.485 \\
1 & -9.9157 & 11.611 & 304.012
\end{array}\right),
$$

$$
C_{2}=\left(\begin{array}{cccc}
720.2154 & -2097.8694 & 652.181 & 14288.789 \\
-2042.393 & -255.0849 & -1199.115 & 20866.450 \\
1 & 0.158828 & -1.34154 & 30.01594
\end{array}\right)
$$

564 The Table 12 shows that the mean values of the space coordinates errors as well as their variances are smaller in case of the Pareto solutions than in case of the implicit method. In addition it is also true for the error vectors 


\section{Conclusions}

We suggested a new method to solve photogrammetric resection-intersection problem. This method based on the explicit formulations of the error of the space as well as the image coordinates leads to a multi-objective optimization problem with competitive objectives. The Pareto solution of this optimization problem provides the user full control to decide which error should be considered to be more important to decrease. In the absence of a decision maker, our method can result in an optimal solution where the residuals for both objectives are smaller than the case of the traditional implicit solution. The illustrative examples indicated that not only the global errors, but also the local errors and their variance can be reduced considerably. Although solving a multi-objective optimization problem requires more computation effort than the single objective problem, employing Levenberg-Marquardt algorithm in parallel way on a multicore processor minimizes this handicap. It should also be mentioned that in contrast to the TLS (total least square) method, this approach allows for the incorporation of both measuring and modelling errors.

\section{Acknowledgements}

The author are grateful to the Editor Prof. Babaie and the anonymous reviewers for their comments that helped improve the quality of the manuscript. Special thanks to Prof. Han for providing their measurements data that was used in one of the examples in this study. They are further grateful to Dr. Kevin Fleming for proof reading the manuscript, but take full responsibility for any errors. J.L. Awange acknowledges the financial support of the 
Alexander von Humboldt Foundation (Ludwig Leichhardt's Memorial Fellowship) and a Curtin Research Fellowship. He is grateful for the warm welcome and the conducive working atmosphere provided by his host, Prof. Bernhard Heck and his team, at the Geodetic Institute, Karlsruhe Institute of Technology (KIT). This is a TiGER publication No. ??

\section{Appendix}

Summary of the steps of the algorithm

Read input data

The coordinates of the points on the photo - planes :

$x_{j}{ }^{(i)}, y_{j}{ }^{(i)}, j=1,2, \ldots n, i=1,2, \ldots m$

The space coordinates :

$X_{j}, Y_{j}, Z_{j}, j=1,2, \ldots n$,

where $n$ is the number of points on a photo - plane, and $m$ is the number of the photo-planes.

Defining the objective functions

a) for the photo - planes:

$$
\begin{aligned}
& G_{\mathrm{xy}}\left(\pi_{1}, \pi_{2}, \ldots \pi_{m}\right)=\sum_{i=1}^{m} \sum_{j=1}^{n} w_{x_{j}}\left(x_{j}{ }^{(i)}-p_{x}\left(\pi_{i}, X_{j}, Y_{j}, Z_{j}\right)\right)^{2}+ \\
& w_{y_{j}}\left(y_{j}{ }^{(i)}-p_{y}\left(\pi_{i}, X_{j}, Y_{j}, Z_{j}\right)\right)^{2}
\end{aligned}
$$

b) for the space coordinates

- use one - point intersection to express the space coordinates explicitely, see Eq.(14):

$$
G_{\mathrm{XYZ}}\left(\pi_{1}, \pi_{2}, \ldots \pi_{m}\right)=\sum_{j=1}^{n} W_{X_{j}}\left(X_{j}{ }^{(i)}-p_{X}\left(\pi_{1}, \pi_{2}, \ldots \pi_{m}, x_{j}{ }^{(i)}, y_{j}{ }^{(i)}\right)\right)^{2}+
$$

$W_{Y_{j}}\left(Y_{j}{ }^{(i)}-p_{Y}\left(\pi_{1}, \pi_{2}, \ldots \pi_{m}, x_{j}{ }^{(i)}, y_{j}{ }^{(i)}\right)\right)^{2}+W_{Z_{j}}\left(Z_{j}{ }^{(i)}-p_{Z}\left(\pi_{1}, \pi_{2}, \ldots \pi_{m}, x_{j}{ }^{(i)}, y_{j}{ }^{(i)}\right)\right)^{2}$ 
- alternatively use implicit expression of the space coordinates as con-

straint while minimizing the adjustments of the space coordinates, see Eq.(20).

$$
G_{\mathrm{XYZ}}\left(\pi_{1}, \pi_{2}, \ldots \pi_{m}\right)=\sum_{j=1}^{n} W_{X_{j}} \Delta \mathrm{X}_{j}^{2}+W_{Y_{j}} \Delta \mathrm{Y}_{j}{ }^{2}+W_{Z_{j}} \Delta \mathrm{Z}_{j}^{2},
$$

with the constraints

$$
\begin{aligned}
& P_{X}\left(\pi_{i}, X_{j}+\Delta \mathrm{X}_{j}, Y_{j}+\Delta \mathrm{Y}_{j}, Z_{j}+\Delta \mathrm{Z}_{j}, x_{j}{ }^{(i)}, y_{j}{ }^{(i)}\right)=0, j=1 . . n \\
& P_{Y}\left(\pi_{i}, X_{j}+\Delta \mathrm{X}_{j}, Y_{j}+\Delta \mathrm{Y}_{j}, Z_{j}+\Delta \mathrm{Z}_{j}, x_{j}{ }^{(i)}, y_{j}{ }^{(i)}\right)=0, j=1 . . n .
\end{aligned}
$$

Computing the dimensionless form of the conflicting objective functions

a) Minimize $G_{\mathrm{xy}}$ to get $\pi_{1}{ }^{(\mathrm{xy})}, \pi_{2}{ }^{(\mathrm{xy})}, \ldots \pi_{m}{ }^{(\mathrm{xy})}$

b) Minimize $G_{\mathrm{XYZ}}$ to get $\pi_{1}{ }^{(\mathrm{XYZ})}, \pi_{2}{ }^{(\mathrm{XYZ})}, \ldots \pi_{m}{ }^{(\mathrm{XYZ})}$

c) The maximum values of the objective functions

$$
G_{\mathrm{xymax}}=G_{\mathrm{xy}}\left(\pi_{1}{ }^{(\mathrm{XYZ})}, \pi_{2}{ }^{(\mathrm{XYZ})}, \ldots \pi_{m}{ }^{(\mathrm{XYZ})}\right)
$$

$G_{\mathrm{XYZmax}}=G_{\mathrm{XYZ}}\left(\pi_{1}{ }^{(\mathrm{xy})}, \pi_{2}{ }^{(\mathrm{xy})}, \ldots \pi_{m}{ }^{(\mathrm{xy})}\right)$

d) Compute the dimensionless forms

$$
\tilde{G}_{\mathrm{xy}}\left(\pi_{1}, \pi_{2}, \ldots \pi_{m}\right)=\frac{G_{\mathrm{xy}}\left(\pi_{1}, \pi_{2}, \ldots \pi_{m}\right)-G_{\mathrm{xymin}}}{G_{\mathrm{xymax}}-G_{\mathrm{xymin}}}
$$

and

$$
\tilde{G}_{\mathrm{XYZ}}\left(\pi_{1}, \pi_{2}, \ldots \pi_{m}\right)=\frac{G_{\mathrm{XYZ}}\left(\pi_{1}, \pi_{2}, \ldots \pi_{m}\right)-G_{\mathrm{XYZ} \min }}{G_{\mathrm{XYZmax}}-G_{\mathrm{XYZ} \min }}
$$

\section{Computing the Pareto set}
a) Set discrete values $\lambda_{k} \in[0,1], \quad k=1,2, \ldots \mathrm{N}$

b) Minimize the mono-objective function for all $\lambda_{k}$ $\tilde{G}\left(\pi_{1}, \pi_{2}, \ldots \pi_{m}, \lambda_{k}\right)=\lambda_{k} \tilde{G}_{\mathrm{XYZ}}\left(\pi_{1}, \pi_{2}, \ldots \pi_{m}\right)+\left(1-\lambda_{k}\right) \tilde{G}_{\mathrm{xy}}\left(\pi_{1}, \pi_{2}, \ldots \pi_{m}\right)$ to get the Pareto-set of $\left(\lambda_{k}, \pi_{1}{ }^{(k)}, \pi_{2}{ }^{(k)}, \ldots \pi_{m}{ }^{(k)}\right), \quad k=1,2, \ldots \mathrm{N}$ 
Computing the Pareto front

a) Set the interpolation functions: $\pi_{1}(\lambda), \pi_{2}(\lambda) \ldots \pi_{m}(\lambda)$ from the discrete values

b) Substitute these functions into the objective functions,

$$
\tilde{G}_{\mathrm{xy}}(\lambda)=\tilde{G}_{\mathrm{xy}}\left(\pi_{1}(\lambda), \pi_{2}(\lambda), \ldots \pi_{m}(\lambda)\right)
$$

and

$\tilde{G}_{\mathrm{XYZ}}(\lambda)=\tilde{G}_{\mathrm{XYZ}}\left(\pi_{1}(\lambda), \pi_{2}(\lambda), \ldots \pi_{m}(\lambda)\right)$

Then we get the Pareto-front represented in parametric form: $\tilde{G}_{\mathrm{xy}}(\lambda)$ $\tilde{G}_{\mathrm{XYZ}}(\lambda)$

\section{Selecting a single solution}

a) $\lambda=0$

we get $\tilde{G}_{\text {min }}=\tilde{G}_{\text {xymin }}$ therefore the point of the Pareto-front for $\lambda=$ 0 is $\left(\tilde{G}_{\text {XYZmax }}, \tilde{G}_{\text {xymin }}\right)$.
b) $\lambda=1$

we get $\tilde{G}_{\min }=\tilde{G}_{\mathrm{XYZmin}}$ therefore the point of the Pareto-frontfront for $\lambda=1$ is $\left(\tilde{G}_{\text {XYZmin }}, \tilde{G}_{\text {xymax }}\right)$.

Consequently to minimize the error of the coordinates of the photo-planes we should select a point of the Pareto-front represented by the parameter $\lambda^{*}$ $<<1$, and vica versa to minimize the error of the space coordinates one should select a point of the Pareto-front with $\lambda^{*}>>0$.

This is therefore a trade-off job for the decision maker.

c) compute the camera parameters $\pi_{i}{ }^{*}$ employing the selected $\lambda^{*}$ as $\pi_{i}^{*}\left(\lambda^{*}\right)$ for $\mathrm{i}=1,2, \ldots \mathrm{m}$. 
Selecting the Pareto-balanced solution

This solution can minimize the overall errors of the coordinates of photoplanes as well as the space coordinates. The point of the Pareto-front representing this solution is the closest point to the ideal point $(0,0)$, which represents zero error for $\tilde{G}_{\mathrm{xy}}$ as well as for $\tilde{G}_{\mathrm{XYZ}}$.

a) use $L_{1}$ norm

$$
\min _{\lambda} \tilde{G}_{\mathrm{xy}}(\lambda)+\tilde{G}_{\mathrm{XYZ}}(\lambda) \longrightarrow \lambda^{*}
$$

b) alternatively use $L_{2}$ norm

$$
\min _{\lambda} \sqrt{\left(\left(\tilde{G}_{\mathrm{xy}}(\lambda)\right)^{2}+\left(\tilde{G}_{\mathrm{XYZ}}(\lambda)\right)^{2}\right.} \longrightarrow \lambda^{*}
$$

\section{References}

Ameller MA, Triggs B, Quan L (2000) Camera pose revisited - New linear algorithms. European Conference on Computer Vision (ECCV).

Atkinson KB (1996) Close range photogrammetry and machine vision. Whittles Publishers.

Awange JL, Kiema JBK (2013) Environmental Geoinformatics - monitoring and management. Springer, Berlin

Awange JL, Grafarend EW (2005) Solving Algebraic Computational Problems in Geodesy and Geoinformatics. Springer, Berlin

Awange JL, Grafarend EW, Paláncz B, Zaletnyik P (2010) Algebraic Geodesy and Geoinformatics. Springer, Berlin. 
Bartoli A (2002) A Unified Framework for Quasi-Linear Bundle Adjustment. ICPR02 - In proceedings of the sixteenth IAPR International Conference on Pattern Recognition, Quebec City, Canada, pp. 560-563, Vol. Ii, August 2002.

Börlin N (2002) Comparison of resection: Intersection algorithms and projection geometries in radiostereometry. ISPRS Journal of Photogrammetry \& Remote Sensing 56: 390 400, doi:10.1016/S0924-2716(02)000680 .

Censor Y (1977) Pareto optimality in multiobjective problems. Applied Mathematics and Optimization 4: 41- 59.

Chen Q, Medioni G (1999) Efficient iterative solution to M-view projective reconstruction. In: Computer Vision and Pattern Recognition, 1999. IEEE Computer Society Conference, doi: 10.1109/CVPR.1999.784608.

Coello CA (1999) A comprehensive survey of evolutionary-based multiobjective optimization techniques. Knowledge and Information Systems 1 (3): 269-308.

Dunn, E., Olague, G., Lutton, E., and Schoenauer, M. (2004) Pareto optimal sensing strategies for an active vision system. IEEE Congress on Evolutionary Computation. Vol. 1, pp. 457-463, Portland, Oregon, USA, June 19-23, 2004.

Fekete K, Schrott P (2008) Qualification of optical capturing devices for data gathering phase of the face reconstruction process. Proc. of 
the Third Hungarian Conference on Biomechanics, Budapest, Hungary, pp.83-88.

Felus YA, Schaffrin B (2005) Performing similarity transformations using the errors-in-variable model. ASPRS Ann. Conference, Baltimore, Maryland.

Forsyth DA, Ponce J (2003) Computer vision - A modern approach. Pearson Education.

Geisler J, Trächtler A (2009) Control of the Pareto optimality of systems with unknown disturbances. IEEE International Conference on Control and Automation Christchurch, New Zealand, December 9-11: 695-700.

Golub GH, van Loan CF (1980) An analysis of the total least-squares problem. SIAM J Numer Anal 17(6):883893.

Grafarend E, Awange JL (2012) Applications of linear and nonlinear models. Springer, New York, Berlin.

Grafarend E, Shan J (1997a) Closed form solution to the P4P or the three dimensional resection problem in terms of Möbius barycentric coordinates. Journal of Geodesy 71: 217-231, doi: 10.1007/s001900050089.

Grafarend E, Shan J (1997b) Closed form solution to the twin P4P or the combined three dimensional resection-intersection problem in terms of Möbius barycentric coordinates. Journal of Geodesy 71 (4): 232-239, doi: $10.1007 / \mathrm{s} 001900050090$. 
Grafarend E, Shan J (1997c) Estimable quantities in projective geometry I and II. Zeitschrift fuer Vermessungswessen, 122 (Heft 5 and 7): 218225, 323-333.

Grussenmeyer P, Al Khalil O (2002) Solution of exterior orientation in photogrammetry, a review. The Photogrammetric Record, An International Journal of Photogrammetry 17 (100): 615-634, doi: 10.1111/j.14779730.2002.tb01907.x.

Han JY, Guo J, Chou JY (2011) A direct determination of the orientation parameters in the collinearity equations. IEEE Geoscience and Remote Sensing Letters 8: 313-316, 10.1109/LGRS.2010.2066955.

Hartley R, Zisserman A (2003) Multiple view geometry in computer vision. Cambridge University Press.

Hochman HM, Rodgers JD (1969) Pareto optimal redistribution. The American Economic Review 59(4): Part 1, pp. 542-557

Lin JG (1976) Multiple-objective problems - Pareto-optimal solutions by method of proper equality constraints. IEEE Transactions on Automatic Control, AC-21: 641-650.

Marler RT, Arora JS (2004) Survey of multi-objective optimization methods for engineering. Struct. Multidisc. Optim. 26: 369395.

McGlone JC (1989) Analytic data-reduction schemes in non-topographic photogrammetry. In: American Society of Photogrammetry and Remote Sensing, Chapter 4. Falls Church, Virginia 554, pp. 37-55. 
Mikhail EM, Bethel JS, McGlone CJ (2001) Introduction to modern photogrammetry. Wiley.

Mirza P, Almir K (2010) Pareto-based genetic algorithm in multi-objective geospatial analysis. In: MIPRO, 2010 Proceedings of the 33rd International Convention , 24-28 May 2010 Opatija, Croatia, pp. 680 - 685.

Mahamud S, Herbert M, Omori Y, Ponce J (2001) Provably-convergent iterative methods for projective structure and motion. In: Computer Vision and Pattern Recognition, 2001. CVPR 2001. Proceedings of the 2001 IEEE Computer Society Conference page(s): I-1018 - I-1025 vol.1, doi: 10.1109/CVPR.2001.990642.

Neitzel F (2010) Generalization of total least-squares on example of unweighted and weighted 2D similarity transformation. Journal of Geodesy, 84 (12): 751-762, doi: 10.1007/s00190-010-0408-0doi: 10.1007/s00190010-0408-0.

Olague G, Trujillo L (2012) Interest point detection through multiobjective genetic programming. Applied Soft Computing, 12(8): 2566-2582, doi: 10.1016/j.asoc.2012.03.058.

Olague G, Trujillo L (2011) Evolutionary-computer-assisted design of image operators that detect interest points using genetic programming. Image and Vision Computing 29: 484-498, doi:10.1016/j.imavis.2011.03.004

Olsson C, Martin Byröd, Kahl F (2009) Globally optimal least squares solutions for quasiconvex 1D vision problems. In: A.-B. Salberg, J.Y. Hardeberg, and R. Jenssen (Eds.): SCIA 2009, LNCS 5575, pp. 686695. 
Paláncz B, Awange JL (2012) Application of Pareto optimality to linear models with errors-in-all-variables. Journal of Geodesy 86 (7): 531545, doi: 10.1007/s00190-011-0536-1.

Pressl B, Mader C, Wieser M(2010) User-specific web-based route planning . In: K. Miesenberger et al. (Eds.): ICCHP 2010, Part I, LNCS 6179, pp. 280-287, 2010. Springer-Verlag, Berlin, Heidelberg.

Remondino F (2002) 3-D reconstruction of articulated objects from uncalibrated images. Three-dimensional image capture and application V, SPIE Electronic Imaging, Proc. of SPIE 4661, San Jose, USA, Jan. 2002.

Saadatseresht M, Mansourian A, Taleai M (2009) Evacuation planning using multi-objective evolutionary optimization approach. European Journal of Operational Research 198: 305-314, doi:10.1016/j.ejor.2008.07.032.

Schaffrin B, Snow K (2010) Total Least-Squares regularization of Tykhonov type and an ancient racetrack in Corinth. Linear Algebra and its Applications 432: 2061-2076, doi:10.1016/j.laa.2009.09.014.

Sonnier DL (2010) A Pareto-optimality based routing and wavelength assignment algorithm for WDM networks. Journal of Computing Sciences in Colleges archive 25 (5): 118-123, .

Triggs B, McLauchlan P, Hartley R, Fitzgibbon A (2000) Bundle adjustment - a modern synthesis. In Vision Algorithms: Theory and Practice, 2000, Springer-Verlag, London. 
Werner T, Schaffalitzky F, Zisserman A. (1999) Automated Architecture Reconstruction from Close-range Photogrammetry. In the proceedings of the International CIPA Symposium, 2001. http://www.robots.ox.ac.uk/vgg/publications/. [Accessed on 19/10/2012].

Warr PG (1982) Pareto optimal redistribution and private charity. Journal of Public Economics 19(1): 131-138, doi:10.1016/0047-2727(82)900561.

Wilson PB, Macleod MD (1993) Low implementation cost IIR digital filter design using genetic algorithms. IEE/IEEE workshop on Natural Algorithms in Signal Processing, 1-8.

Young-Hoo Kwon (1998) DLT Method. www.kwon3d.com/theory/dlt/dlt.html [Accessed on 17/11/2011].

Zitler E, Thiele L (1999) Multiobjective Evolutionary algorithms: A comparative case study and the strength of pareto approach. IEEE Transactions On Evolutionary Computation 3(4): 257-271, doi: 10.1109/4235.797969.

Zwanzig S (2006) On an application of deconvolution techniques to local linear regression with errors in variables. Department of Mathematics Uppsala University, U.U.D.M. Report 2006:12. 


\begin{tabular}{|c|c|c|c|c|c|}
\hline$\lambda$ & $G_{X Y Z}$ & $G_{x y u v}$ & $\mathrm{RSME}$ & $\mathrm{RSME}$ & $\tilde{G}=\tilde{G}_{X Y Z}+\tilde{G}_{x y u v}$ \\
$10^{-8}$ & $G_{X Y Z}$ & $\begin{array}{c}G_{x y u v} \\
10^{-5}\end{array}$ & \\
\hline 0 & 0.01382 & 1.22089 & 0.0326025 & 3.06455 & $0+1=1$ \\
\hline 1 & 0.00705 & 48.7398 & 0.0232875 & 19.3629 & $1+0=1$ \\
\hline 0.34 & 0.00723 & 1.92424 & 0.0235795 & 3.84732 & $0.0263+0.0148=0.0411$ \\
\hline
\end{tabular}

811

Table 1: The extreme and the Pareto optimum solutions

Table 2: Camera parameters corresponding to the selected single Pareto optimum solution and the results of Han et al. (2011) for both images.

\section{List of Tables}

\begin{tabular}{|c|c|c|c|c|}
\hline$\cdot$ & $\begin{array}{c}\text { image 1 } \\
\text { Han et al (2011) }\end{array}$ & $\begin{array}{c}\text { image 2 } \\
\text { Han et al (2011) }\end{array}$ & image 1 & image 2 \\
\hline$f$ & 0.188843 & 0.188843 & 0.019101 & 0.019443 \\
\hline$\eta_{0}$ & 0.011899 & 0.011899 & 0.012430 & 0.012703 \\
\hline$\xi_{0}$ & 0.008080 & 0.008080 & 0.008148 & 0.007342 \\
\hline$X_{0}$ & 305206.651 & 305206.651 & 305207.000 & 305213.000 \\
\hline$Y_{0}$ & 2767915.18 & 2767915.18 & 2767915.44 & 2767927.92 \\
\hline$Z_{0}$ & 31.345 & 31.345 & 30.786 & 30.831 \\
\hline$\omega$ & -2.8592 & -2.0151 & -2.8836 & -2.0603 \\
\hline$\varphi$ & -1.2802 & -0.7971 & -1.2256 & -0.7529 \\
\hline$\kappa$ & -2.8419 & -1.9666 & -2.8645 & -2.0005 \\
\hline
\end{tabular}




\begin{tabular}{|c|c|c|c|c|c|c|c|}
\hline Point & $\begin{array}{c}X \\
{[\mathrm{~cm}]}\end{array}$ & $\begin{array}{c}Y \\
{[\mathrm{~cm}]}\end{array}$ & $\begin{array}{c}Z \\
{[\mathrm{~cm}]}\end{array}$ & $\begin{array}{c}x \\
{[\text { pixel] }}\end{array}$ & $\begin{array}{c}y \\
{[\text { pixel] }}\end{array}$ & $\begin{array}{c}u \\
{[\text { pixel] }}\end{array}$ & $\begin{array}{c}v \\
{[\text { pixel] }}\end{array}$ \\
\hline 1 & 37.0928 & 270.932 & 60.5645 & -1904.98 & 1075.32 & -1481.2 & 1180.57 \\
\hline 2 & 155.314 & 270.415 & 70.7968 & -944.874 & 1182.3 & -413.785 & 1190.5 \\
\hline 3 & 186.293 & 270.774 & 29.55 & -513.899 & 1002.2 & -160.768 & 926.867 \\
\hline 4 & 37.2884 & 211.556 & 20.3706 & -1702.67 & 448.357 & -1451.84 & 527.715 \\
\hline 5 & 216.672 & 271.041 & 10.598 & -210.173 & 935.471 & 57.3334 & 818.785 \\
\hline 6 & 276.377 & 271.479 & 40.1353 & 305.651 & 1082.49 & 556.102 & 940.561 \\
\hline 7 & 276.824 & 241.776 & 50.2347 & 303.859 & 852.757 & 614.891 & 803.118 \\
\hline 8 & 336.705 & 211.719 & 30.7482 & 902.528 & 492.02 & 1052.18 & 475.928 \\
\hline 9 & 96.9378 & 122.618 & 56.9734 & -1550.04 & -316.229 & -1107.88 & -104.459 \\
\hline 10 & 96.6709 & 271.279 & 19.8864 & -1204.64 & 934.683 & -876.831 & 913.651 \\
\hline 11 & 126.527 & 270.967 & 31.904 & -1023.43 & 989.818 & -645.626 & 965.759 \\
\hline 12 & 66.573 & 241.361 & 25.873 & -1489.44 & 707.986 & -1168.83 & 759.207 \\
\hline 13 & 186.946 & 240.736 & 15.5808 & -489.126 & 698.11 & -163.128 & 663.198 \\
\hline 14 & 156.746 & 211.534 & 18.9318 & -772.346 & 455.653 & -414.46 & 487.708 \\
\hline 15 & 97.5291 & 182.007 & 34.018 & -1350.6 & 227.194 & -978.389 & 345.786 \\
\hline
\end{tabular}




\begin{tabular}{|c|c|c|c|c|c|c|}
\hline \multirow[b]{2}{*}{ Training set } & \multicolumn{2}{|c|}{$\begin{array}{l}\text { Mean } \\
{[\text { pixel }]}\end{array}$} & \multicolumn{2}{|c|}{$\begin{array}{l}\text { Variance } \\
{\left[\text { pixel }^{2}\right]}\end{array}$} & \multicolumn{2}{|c|}{$\begin{array}{c}\boldsymbol{L}_{2}-\text { norm } \\
{[\text { pixel }]}\end{array}$} \\
\hline & $\Delta x$ & $\Delta y$ & $\Delta x$ & $\Delta y$ & Mean & Variance \\
\hline photo - plane1 & 2.4733 & 3.6567 & 4.9693 & 1.2772 & 4.7363 & 2.9347 \\
\hline photo - plane 2 & 2.38101 & 3.4946 & 3.8441 & 3.8482 & 4.6224 & 3.7715 \\
\hline \multicolumn{7}{|l|}{ Validation set } \\
\hline photo - plane1 & 5.1082 & 2.0776 & 7.5663 & 2.5446 & 5.7148 & 7.4121 \\
\hline photo - plane2 & 5.6771 & 1.8655 & 8.0390 & 1.3439 & 6.3514 & 3.8269 \\
\hline
\end{tabular}


Table 6: Error in the space coordinates (measured-computed).

\begin{tabular}{|c|c|c|c|c|c|c|c|c|}
\hline \multirow[t]{2}{*}{. } & \multicolumn{3}{|c|}{$\begin{array}{c}\text { Mean } \\
{[\mathrm{cm}]}\end{array}$} & \multicolumn{3}{|c|}{$\begin{array}{c}\text { Variance } \\
{\left[\mathrm{cm}^{2}\right]}\end{array}$} & \multicolumn{2}{|c|}{$\begin{array}{c}L_{2}-\text { norm } \\
{[\mathrm{cm}]}\end{array}$} \\
\hline & $\Delta X$ & $\Delta Y$ & $\Delta Z$ & $\Delta X$ & $\Delta Y$ & $\Delta Z$ & Mean & Variance \\
\hline Training set & 0.3107 & 0.2745 & 0.3166 & 0.0548 & 0.0162 & 0.0338 & 0.5765 & 0.0370 \\
\hline Validation set & 0.7403 & 0.3204 & 0.7035 & 0.1132 & 0.0556 & 0.0672 & 1.1133 & 0.1235 \\
\hline
\end{tabular}

Table 7: The parameter values of the optimal Pareto solution $(\lambda=$

\begin{tabular}{cccc}
\hline . & photo - plane & & photo - plane \\
& 1 & & 2 \\
\hline$a$ & 0.0694594 & $a$ & 0.206368 \\
$b$ & 0.0829882 & $b$ & -0.0519138 \\
$c$ & 0.0147064 & $c$ & 0.00269866 \\
$\mathrm{X}_{01}$ & 283.46 & $\mathrm{X}_{02}$ & 168.859 \\
$\mathrm{Y}_{01}$ & 131.854 & $\mathrm{Y}_{02}$ & 41.6848 \\
$\mathrm{Z}_{01}$ & 301.617 & $\mathrm{Z}_{02}$ & 300.968 \\
$\eta_{01}$ & -98.2993 & $\eta_{02}$ & -54.5653 \\
$\xi_{01}$ & 88.39 & $\xi_{02}$ & 112.255 \\
$f_{1}$ & 2696.62 & $f_{2}$ & 2678.54 \\
\hline
\end{tabular}


Table 8: Comparison of the global results of the different solutions

\begin{tabular}{cccccc}
\hline Solution & $\begin{array}{c}G_{\text {XYZ }} \\
{\left[\mathrm{cm}^{2}\right]}\end{array}$ & $\begin{array}{c}G_{\text {xyuv }} \\
{\left[\text { pixel }^{2}\right]}\end{array}$ & $\tilde{G}_{\text {XYZ }}$ & $\tilde{G}_{\text {xyuv }}$ & $\tilde{G}$ \\
\hline Traditional solution & 3.29327 & 447.842 & 1.21375 & 0.00014471 & 1.21389 \\
Pareto balanced & 1.79202 & 1570.96 & 0.0178549 & 0.0400972 & 0.0579521 \\
Selected single optimum & $\mathbf{2 . 4 4 1 5 2}$ & $\mathbf{4 4 7 . 8 1 7}$ & $\mathbf{0 . 5 3 5 2 4 5}$ & $\mathbf{0 . 0 0 0 1 4 3 8 0 8}$ & $\mathbf{0 . 5 3 5 3 8 9}$ \\
Ideal minimum & 1.76961 & 443.774 & 0 & 0 & 1 \\
Ideal maximum & 3.02495 & 28555.1 & 1 & 1 & 1 \\
\hline
\end{tabular}

Table 9: Statistics of $L_{2}$-norm of the local error vectors

\begin{tabular}{ccccc}
\hline & \multicolumn{2}{c}{ Traditional solution } & \multicolumn{2}{c}{ Selected single } \\
& & & \multicolumn{2}{c}{ Pareto optimum } \\
& Mean & Variance & Mean & Variance \\
\hline Training set & & & &. \\
photo - plane1 & 4.7363 & 2.9347 & 4.7700 & 2.6275 \\
photo - plane2 & 4.6224 & 3.7715 & 4.5871 & 4.0813 \\
space coordinates & $\mathbf{0 . 5 7 6 5}$ & $\mathbf{0 . 0 3 7 0}$ & $\mathbf{0 . 4 9 8 2}$ & $\mathbf{0 . 0 2 6 0}$ \\
Validation set & &. & & \\
photo - plane1 & 5.7148 & 7.4121 & 6.0545 & 6.9435 \\
photo - plane2 & 6.3514 & 3.8269 & 5.9766 & 2.9984 \\
space coordinates & $\mathbf{1 . 1 1 3 3}$ & $\mathbf{0 . 1 2 3 6}$ & $\mathbf{0 . 9 8 7 1}$ & $\mathbf{0 . 0 5 5 2}$ \\
\hline
\end{tabular}

Table 10: Image and space coordinates of the points appearing in Fig. 9 
(Source: Werner et al. 1999).

\begin{tabular}{cccccccc}
\hline Point & $x[$ pixel $]$ & $y[$ pixel $]$ & $u[$ pixel $]$ & $v[$ pixel $]$ & $X[m]$ & $Y[m]$ & $Z[m]$ \\
\hline 1 & 705.999 & 98.9828 & 745.015 & 107.986 & 6.66074 & -0.60789 & 4.15341 \\
2 & 537.06 & 243.164 & 565.024 & 278.734 & 4.57591 & -0.314284 & 0.381324 \\
3 & 886.637 & 352.008 & 938.827 & 416.001 & 2.10037 & -0.205085 & 7.35645 \\
4 & 274.06 & 55.0357 & 255.239 & 80.2127 & 7.28601 & 5.14973 & -0.395317 \\
5 & 1020.15 & 146.064 & 1020.12 & 170.596 & 4.71216 & 0.165413 & 9.40504 \\
6 & 351.763 & 332.001 & 337.832 & 366.963 & 2.87013 & 3.88323 & 0.0713809 \\
7 & 595.754 & 127.136 & 631.718 & 143.193 & 6.69286 & -0.693863 & 1.67512 \\
8 & 427.277 & 175.001 & 447.456 & 203.001 & 5.86426 & 1.40346 & -0.735147 \\
9 & 240.998 & 377.619 & 203.998 & 434.228 & 1.81334 & 6.00578 & 0.0141997 \\
10 & 691.011 & 347.1 & 722.031 & 401.938 & 2.33644 & -0.150519 & 4.07266 \\
11 & 296.71 & 92.038 & 283.676 & 117.139 & 6.75543 & 4.66651 & -0.419889 \\
12 & 168.112 & 214.997 & 119.784 & 252.987 & 4.30466 & 7.29488 & -0.0117434 \\
13 & 698.692 & 155. & 740.268 & 174. & 5.80751 & -0.925267 & 3.76603 \\
14 & 765.159 & 445.002 & 801.956 & 521.002 & 0.732124 & -0.382526 & 5.24415 \\
15 & 694.685 & 119. & 736.68 & 132.002 & 6.47018 & -0.910284 & 3.70673 \\
16 & 2.98298 & 128.5 & 2.96668 & 181.091 & 5.03421 & 9.90634 & 0.316602 \\
17 & 830.993 & 128.003 & 884.692 & 138.002 & 5.77407 & -0.855155 & 6.26391 \\
18 & 604.007 & 238.555 & 635.01 & 273.123 & 4.45486 & -0.350295 & 2.11096 \\
19 & 735.994 & 305.993 & 769.558 & 354.002 & 2.97984 & -0.156468 & 4.9221 \\
20 & 842.392 & 63.6569 & 898.512 & 63.4244 & 6.53113 & -0.553974 & 6.72735 \\
21 & 737.164 & 359.996 & 770.001 & 419.999 & 2.08665 & -0.150263 & 4.91769 \\
22 & 590.272 & 178.001 & 630.331 & 203. & 5.8271 & -1.02223 & 1.2605 \\
23 & 899.698 & 434.012 & 955.923 & 520.004 & 0.883655 & -0.387393 & 7.42723 \\
24 & 110.061 & 281.037 & 45.0181 & 330.655 & 3.15523 & 8.21419 & 0.0342828 \\
25 & 713.818 & 285.001 & 748.573 & 368.005 & 3.37495 & -0.375693 & 4.36912 \\
\hline & & & & & & & \\
\hline
\end{tabular}


Table 11: Comparison of the global results of the different solutions

\begin{tabular}{cccccc}
\hline Solution & $\begin{array}{c}G_{\text {XYZ }} \\
{\left[\mathrm{m}^{2}\right]}\end{array}$ & $\begin{array}{c}G_{\text {xyuv }} \\
{\left[\mathrm{pixel}^{2}\right]}\end{array}$ & $\tilde{G}_{\text {XYZ }}$ & $\tilde{G}_{\text {xyuv }}$ & $\tilde{G}$ \\
\hline Implicit solution & 52.787 & 7671.0 & 1.24279 & 0.002040 & 1.24483 \\
Pareto balanced & 2.26596 & $\mathbf{4 2 0 9 8 . 5}$ & $\mathbf{0 . 0 2 4 6 8 5}$ & $\mathbf{0 . 0 1 6 7 4 7 5}$ & $\mathbf{0 . 0 4 1 4 3 2 5}$ \\
Pareto optimum & & & & & \\
$\lambda=0.1$ & $\mathbf{1 . 7 9 3 0 8}$ & $\mathbf{1 1 3 0 1 0 .}$ & $\mathbf{0 . 0 1 3 2 8}$ & $\mathbf{0 . 0 4 7 0 4 1}$ & $\mathbf{0 . 0 6 0 3 2 1}$ \\
Minimum & $\mathbf{1 . 2 4 2 1}$ & $\mathbf{2 8 9 5 . 6 2}$ & $\mathbf{0}$ & $\mathbf{0}$ & - \\
Maximum & $\mathbf{4 2 . 7 1 7}$ & $\mathbf{2 . 3 4 3 7 2 \times \mathbf { 1 0 }}$ & $\mathbf{1}$ & $\mathbf{1}$ & - \\
\hline
\end{tabular}

Table 12: Comparison of the statistics of the local results of the different solutions, where $\Delta=(\Delta \mathrm{X}, \Delta \mathrm{Y}, \Delta \mathrm{Z})^{T}$ is the error vector of the space coordinates.

\begin{tabular}{cccc}
\hline Solution & Implicit & Pareto balanced & $\begin{array}{c}\text { Selected single } \\
\text { Pareto optimal } \\
\lambda=0.1\end{array}$ \\
\hline$M(\Delta \mathrm{X}),[m]$ & 0.030154 & 0.0039071 & 0.0000549849 \\
$M(\Delta \mathrm{Y}),[m]$ & -0.0138212 & -0.011871 & -0.00231642 \\
$M(\Delta \mathrm{Z}),[m]$ & -0.0671351 & 0.00351096 & -0.000160302 \\
$\sigma^{2}(\Delta \mathrm{X}),\left[m^{2}\right]$ & 0.0609208 & 0.00923592 & 0.00706027 \\
$\sigma^{2}(\Delta \mathrm{Y}),\left[m^{2}\right]$ & 0.739865 & 0.0473309 & 0.0411554 \\
$\sigma^{2}(\Delta \mathrm{Z}),\left[m^{2}\right]$ & 1.39283 & 0.0376726 & 0.0264903 \\
$M\left(L_{2}-\mathrm{norm}(\Delta)\right),[m]$ & 0.93245 & 0.259098 & 0.235654 \\
$\sigma^{2}\left(L_{2}-\operatorname{norm}(\Delta)\right),\left[m^{2}\right]$ & 1.29377 & 0.0244858 & 0.0168648 \\
\hline
\end{tabular}




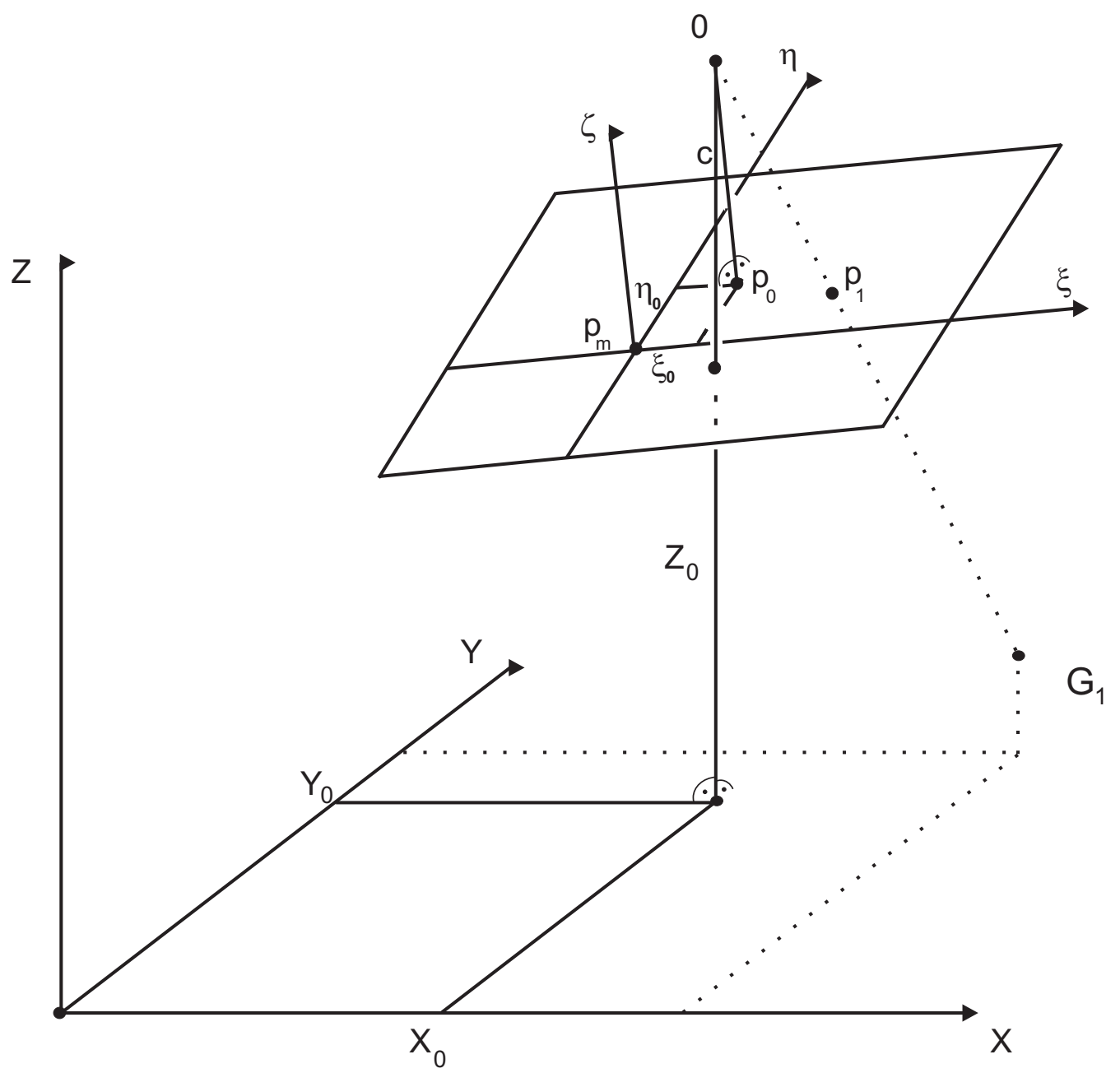

Figure 1: Orientation of the photo space with respect to the object space. $\left\{\xi_{O}, \eta_{O}, c\right\}$ define elements of interior orientation while $\left\{X_{O}, Y_{O}, Z_{O}\right\}$ are part of the exterior orientation elements besides the rotation elements 


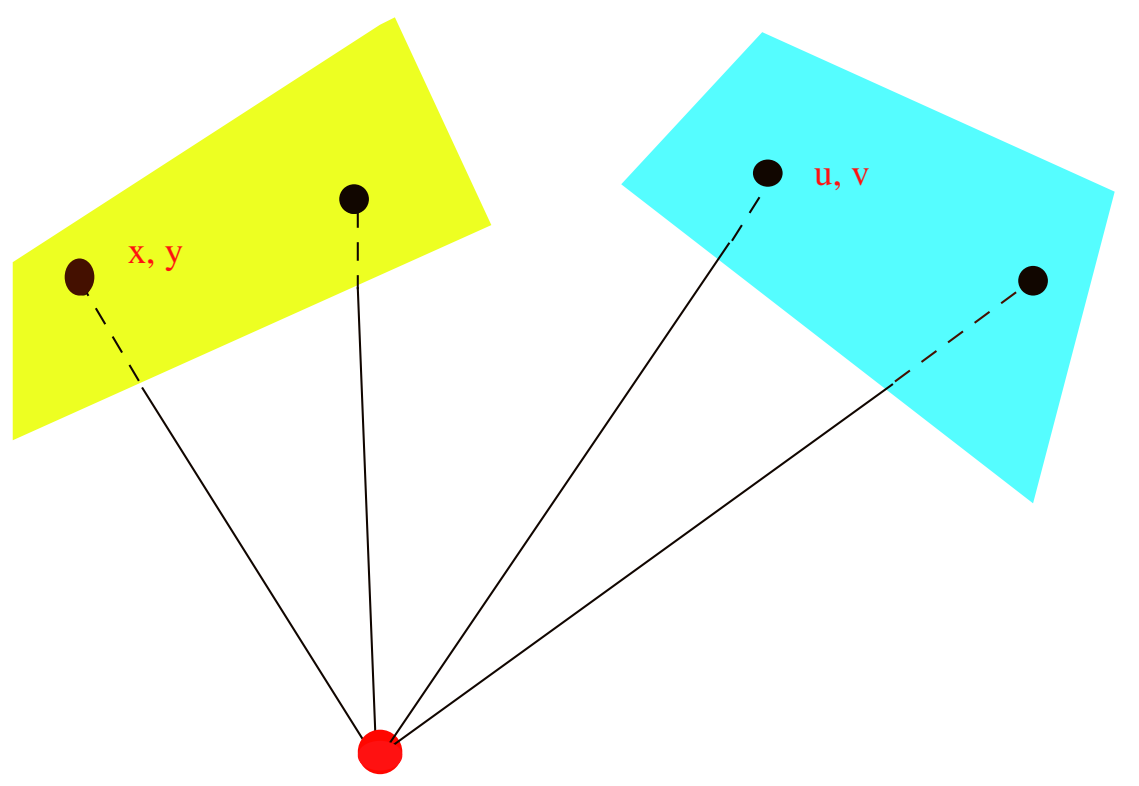

$\mathrm{X}, \mathrm{Y}, \mathrm{Z}$

Figure 2: Photogrammetric 3D intersection. $x, y$ are the image coordinates of the left photo while $u, v$ are the corresponding coordinates of the same image on the right photo. $X, Y, Z$ provides the corresponding coordinates in the object space 


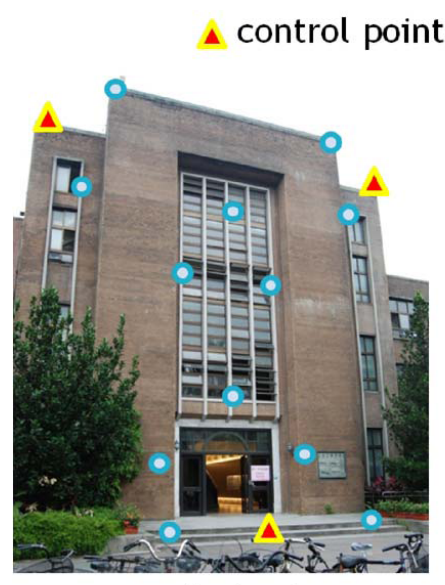

Station 1 o check point

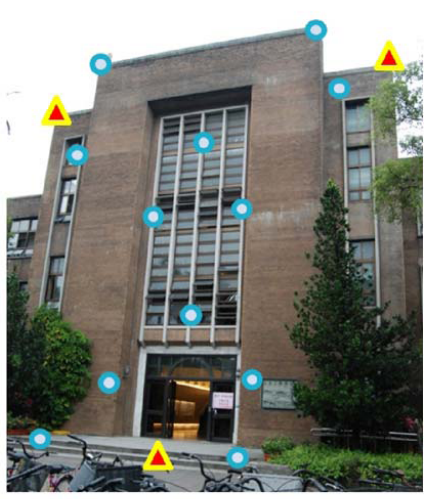

Station 2

Figure 3: The control and check points of images acquired at the two camera stations (the figure adopted from Han et al. (2011). 


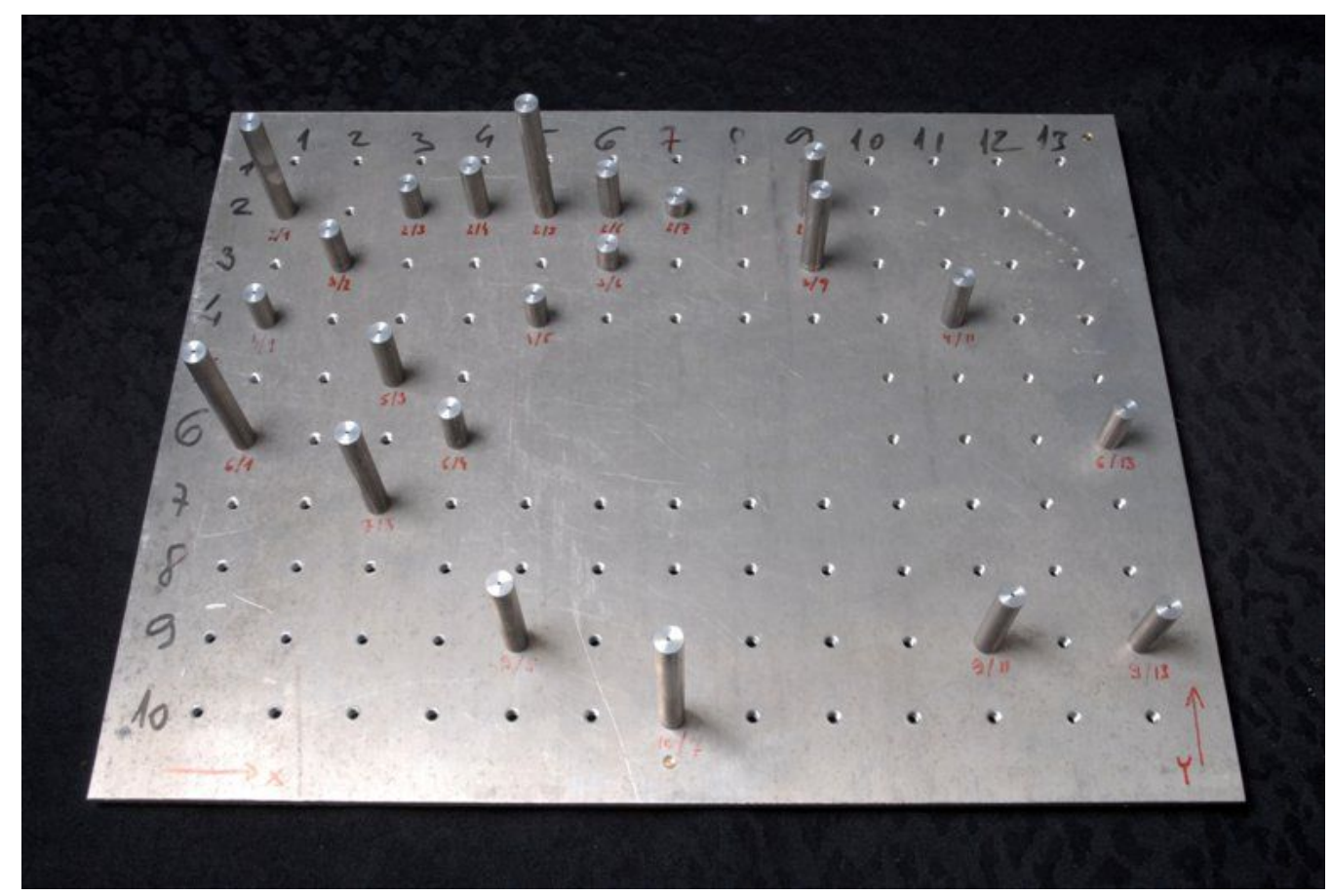

Figure 4: The general Manhattan test field with 22 points. The example in this work used only 15 points for evaluation since the other data points were corrupted. 

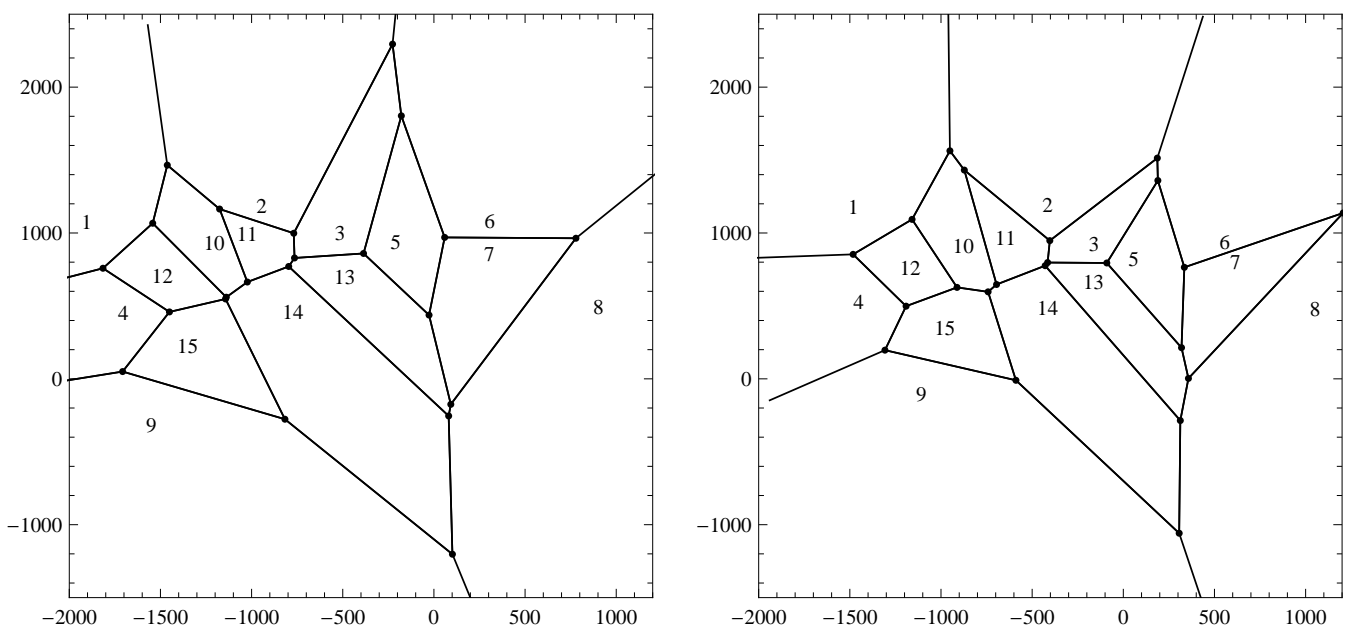

Figure 5: The Voronoi-cells of the training $(1-9)$ and the validation points (10-15) on two photo planes.

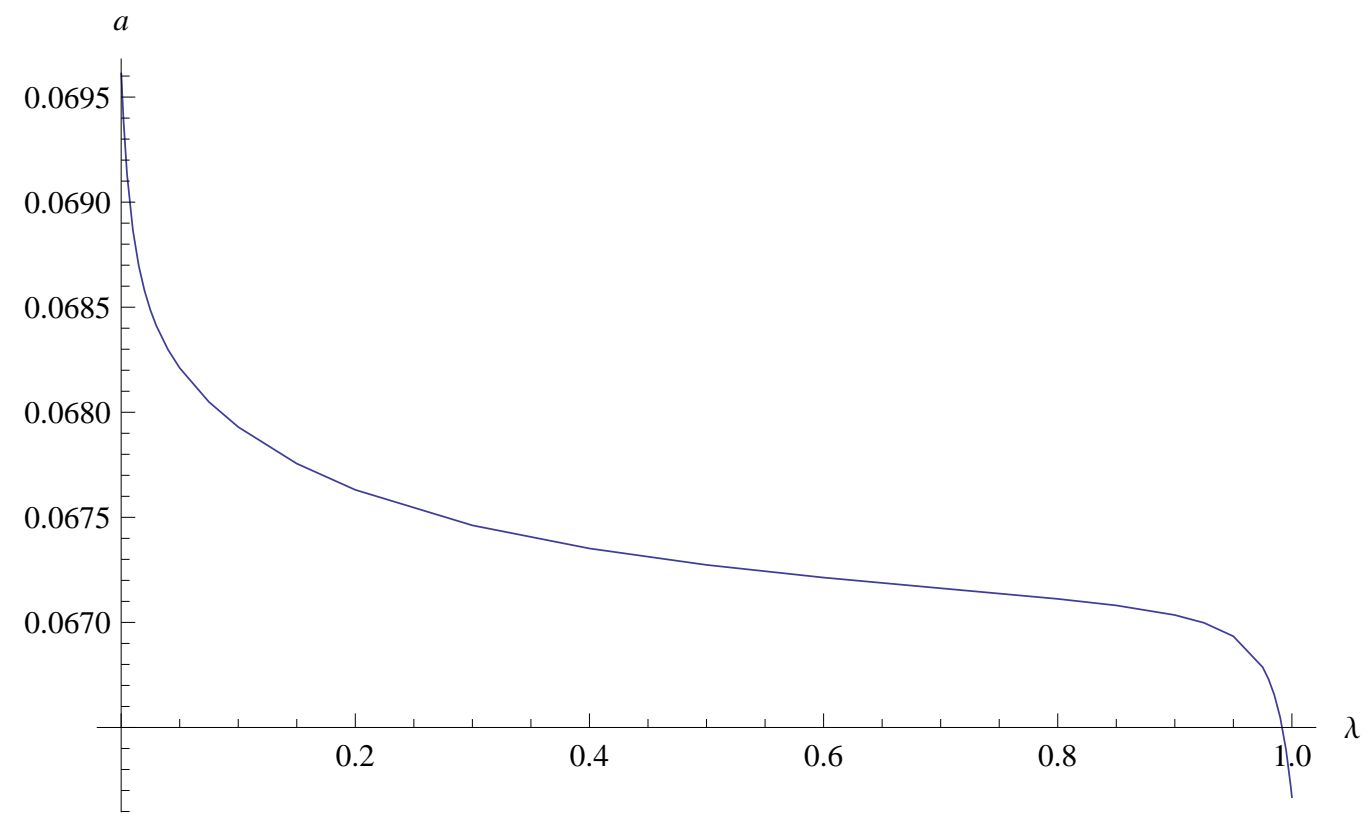

Figure 6: The parameter (a) of the skew matrix $S$ as function of $\lambda$. 


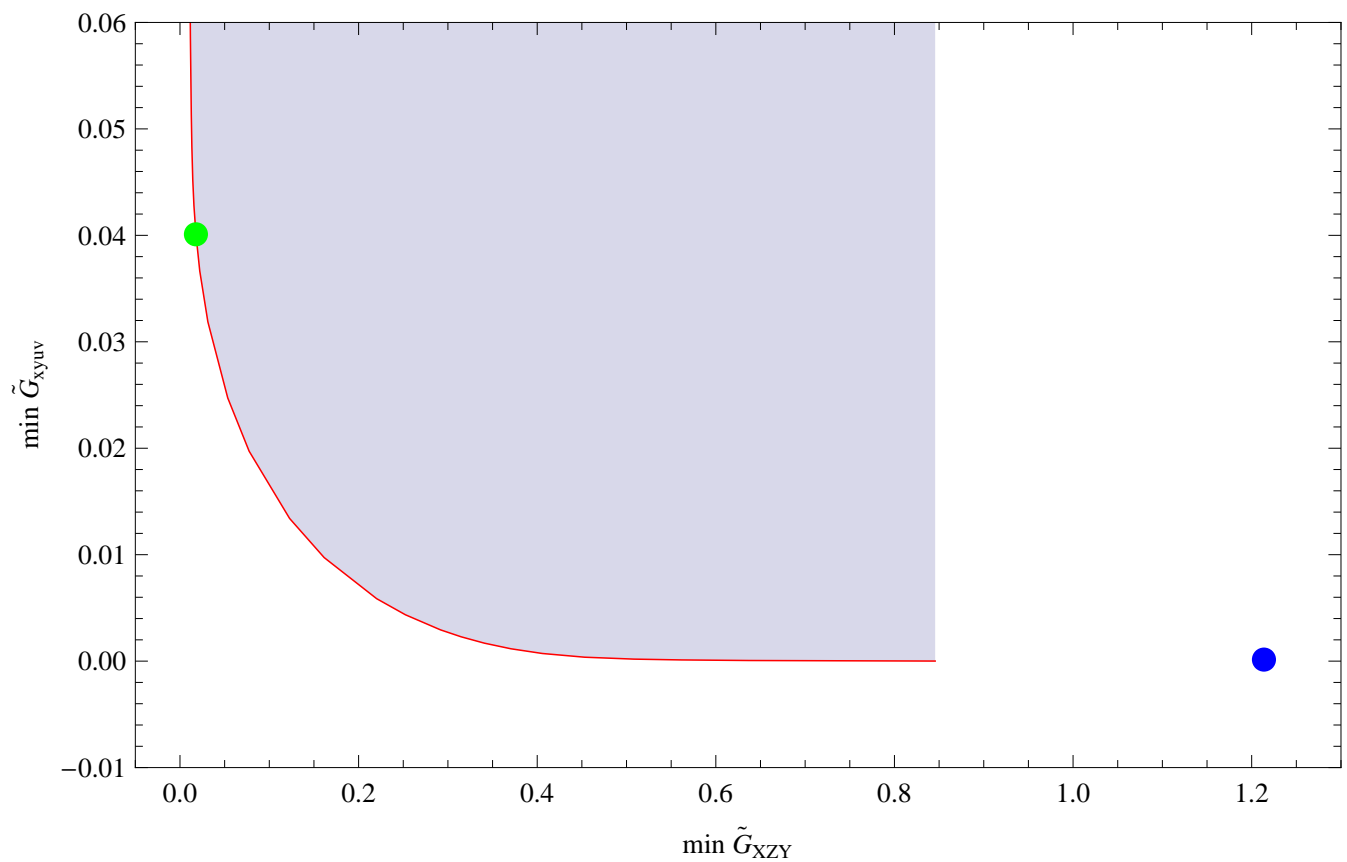

Figure 7: The Pareto-front with the Pareto balanced optimum (green). The results of the traditional method is shown in blue. 


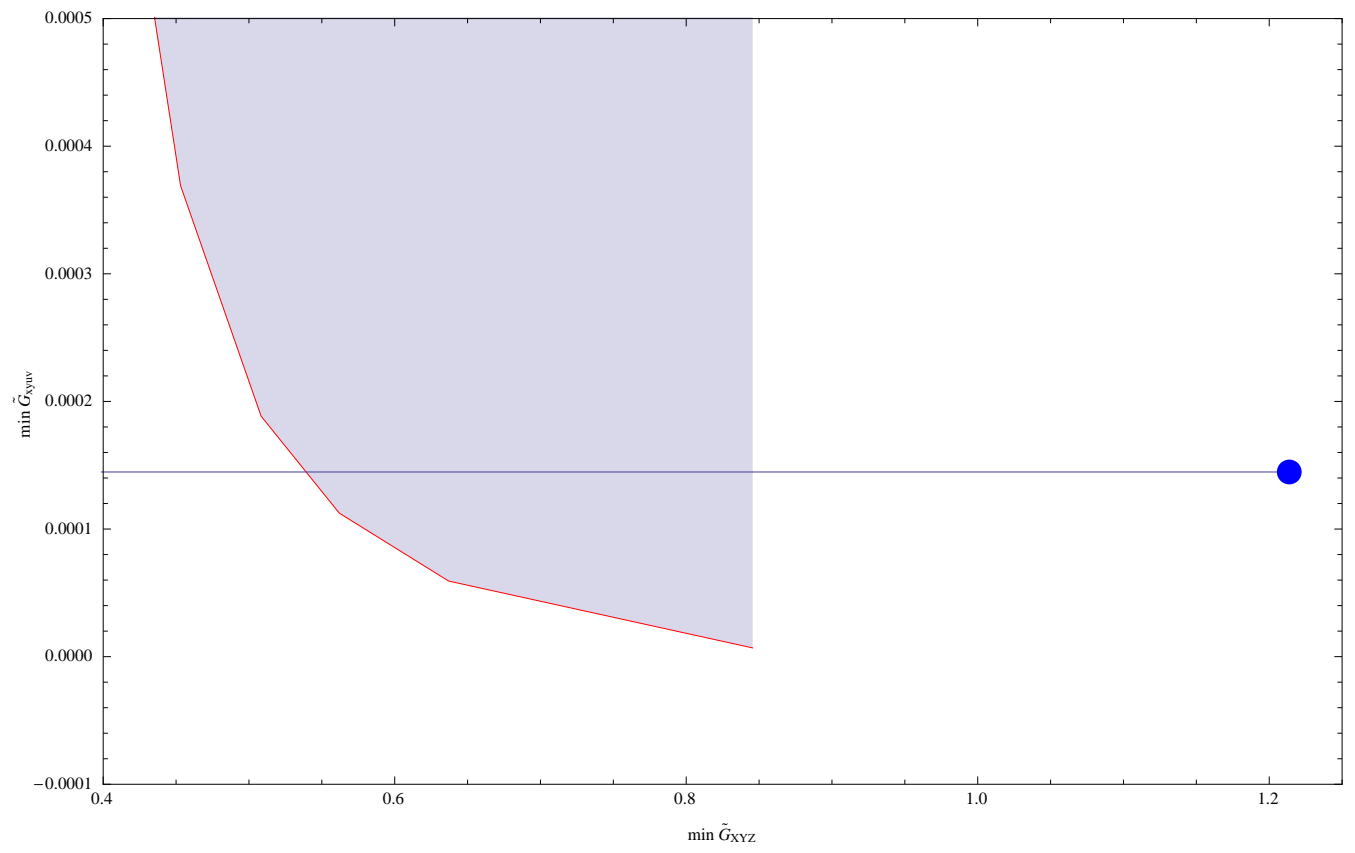

Figure 8: The optimum solution of the Pareto-front, which provides smaller residual for both objectives than those of the traditional solution (red points under the blue line).
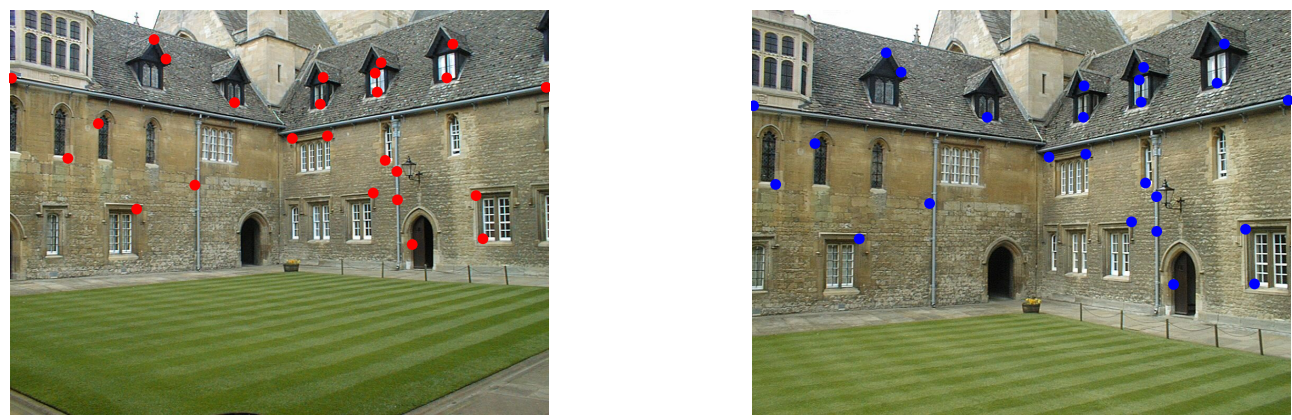

Figure 9: Left: The first photo-plane of Merton College, Oxford, with the data points in red. Right: the second photo-plane of Merton College, Oxford, with the data points in blue. 


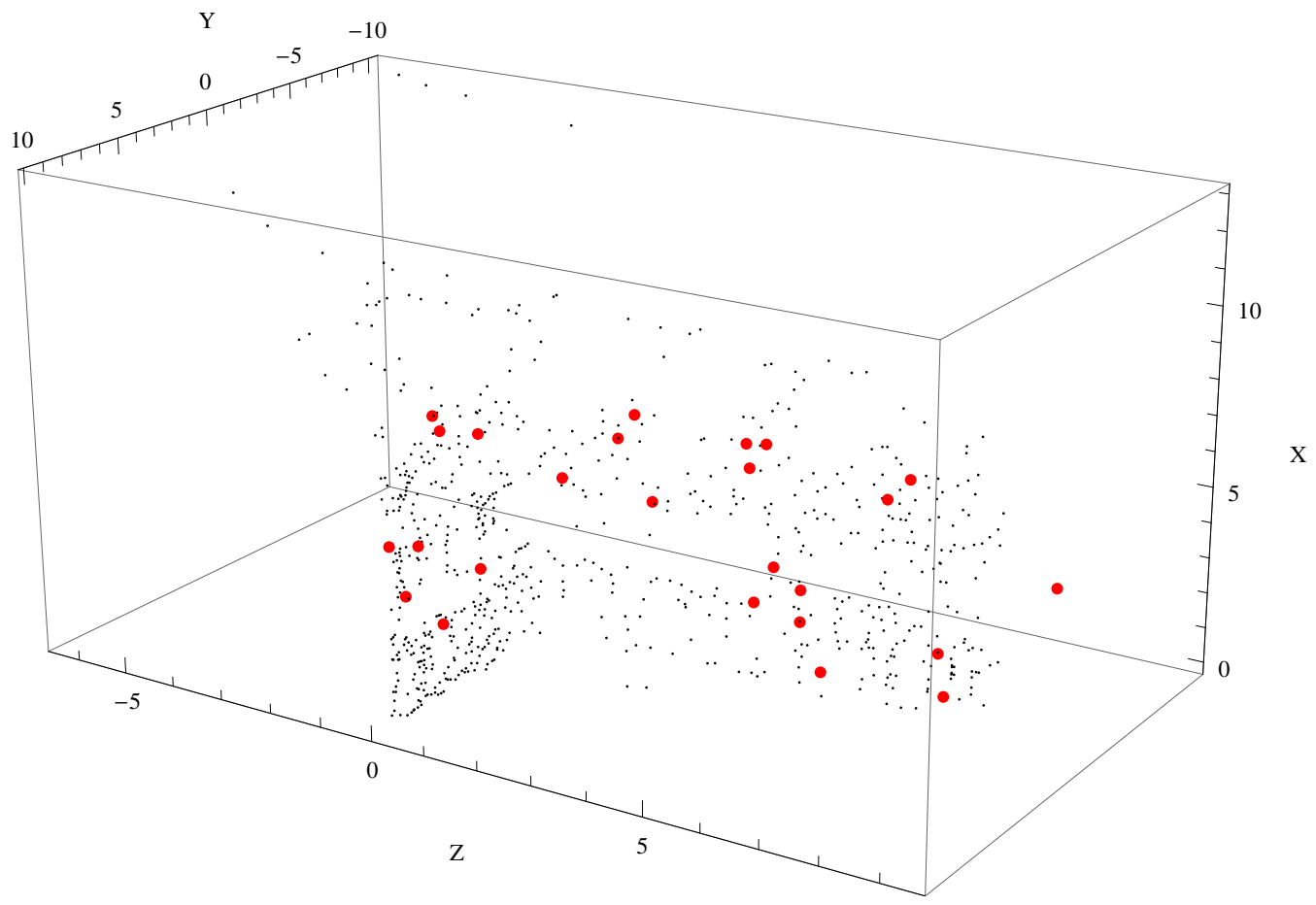

Figure 10: The Merton College's data points in 3D with the cloud-point model as background. 


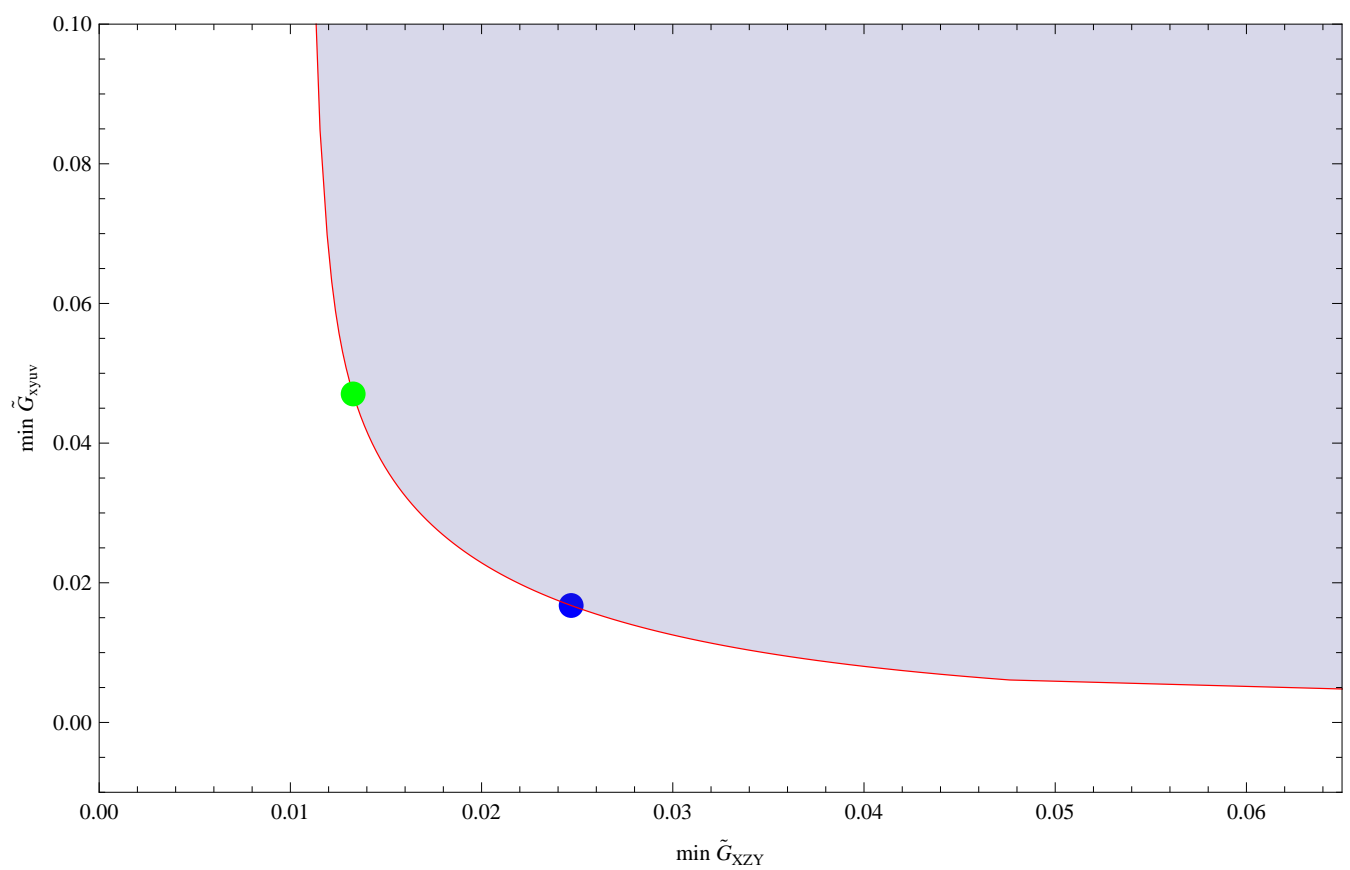

Figure 11: The Pareto-front with the Pareto balanced solution (blue point) and Pareto optimum solution (green point) for the architectural reconstruction. 